\title{
THE XMM-NEWTON WIDE FIELD SURVEY IN THE COSMOS FIELD: REDSHIFT EVOLUTION OF AGN BIAS AND SUBDOMINANT ROLE OF MERGERS IN TRIGGERING MODERATE-LUMINOSITY AGNs AT REDSHIFTS UP TO 2.2
}

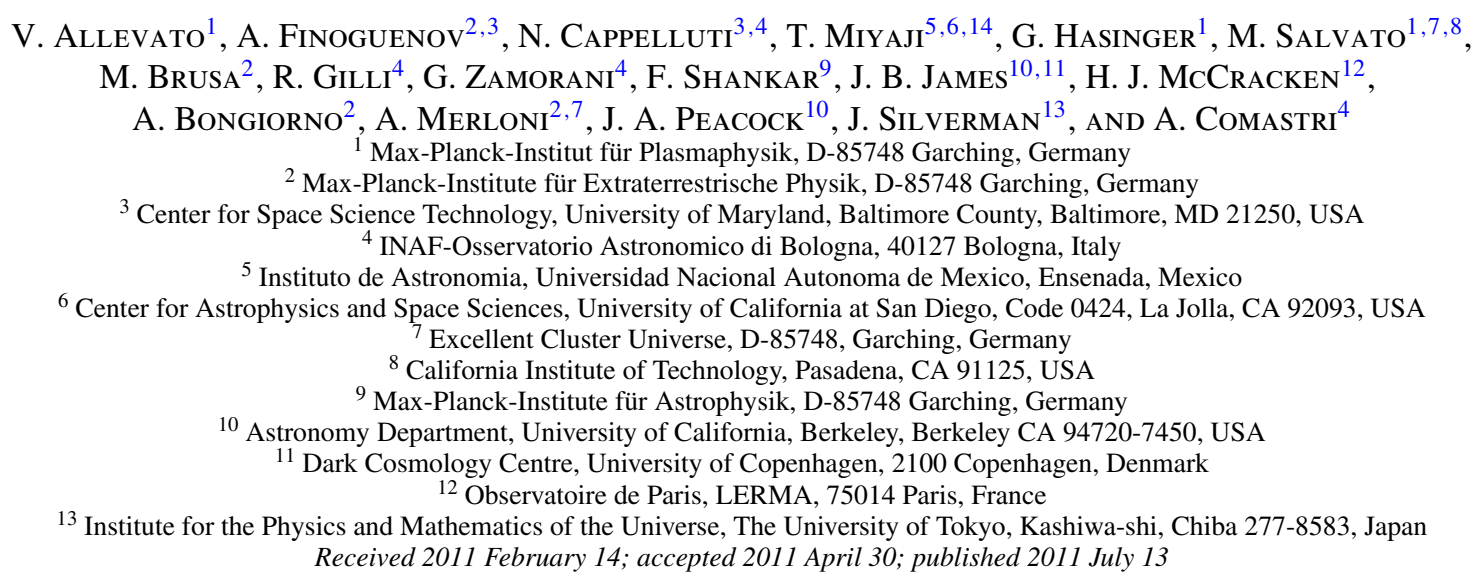

\begin{abstract}
We present a study of the redshift evolution of the projected correlation function of $593 \mathrm{X}$-ray selected active galactic nuclei (AGNs) with $I_{\mathrm{AB}}<23$ and spectroscopic redshifts $z<4$, extracted from the $0.5-2 \mathrm{keV} \mathrm{X-ray} \mathrm{mosaic} \mathrm{of}$ the $2.13 \mathrm{deg}^{2} X M M$ - Cosmic Evolution Survey (COSMOS). We introduce a method to estimate the average bias of the AGN sample and the mass of AGN hosting halos, solving the sample variance using the halo model and taking into account the growth of the structure over time. We find evidence of a redshift evolution of the bias factor for the total population of XMM-COSMOS AGNs from $\bar{b}(\bar{z}=0.92)=2.30 \pm 0.11$ to $\bar{b}(\bar{z}=1.94)=4.37 \pm 0.27$ with an average mass of the hosting dark matter $(\mathrm{DM})$ halos $\log M_{0}\left(h^{-1} M_{\odot}\right) \sim 13.12 \pm 0.12$ that remains constant at all $z<2$. Splitting our sample into broad optical line AGNs (BL), AGNs without broad optical lines $(\mathrm{NL})$, and X-ray unobscured and obscured AGNs, we observe an increase of the bias with redshift in the range $\bar{z}=0.7-2.25$ and $\bar{z}=0.6-1.5$ which corresponds to a constant halo mass of $\log M_{0}\left(h^{-1} M_{\odot}\right) \sim 13.28 \pm 0.07$ and $\log M_{0}\left(h^{-1} M_{\odot}\right) \sim 13.00 \pm 0.06$ for BL/X-ray unobscured AGNs and NL/X-ray obscured AGNs, respectively. The theoretical models, which assume a quasar phase triggered by major mergers, cannot reproduce the high bias factors and DM halo masses found for X-ray selected BL AGNs with $L_{\mathrm{BOL}} \sim 2 \times 10^{45} \mathrm{erg} \mathrm{s}^{-1}$. Our work extends up to $z \sim 2.2$ the $z \lesssim 1$ statement that, for moderate-luminosity X-ray selected BL AGNs, the contribution from major mergers is outnumbered by other processes, possibly secular ones such as tidal disruptions or disk instabilities.
\end{abstract}

Key words: dark matter - galaxies: active - galaxies: evolution - large-scale structure of universe - quasars: general - surveys

Online-only material: color figures

\section{INTRODUCTION}

Investigating the clustering properties of active galactic nuclei (AGNs) is important to put tight constraints on how the AGNs are triggered and fueled, to identify the properties of the AGN host galaxies, and to understand how galaxies and AGNs coevolve. In addition, in the framework of the cold dark matter (CDM) structure formation scenario, clustering properties or the bias of AGNs may be related to the typical mass of dark matter (DM) halos in which they reside (Mo \& White 1996; Sheth \& Tormen 1999; Sheth et al. 2001; Tinker et al. 2005) and allow various types of AGNs to be placed in a cosmological context.

Recently, several studies have been conducted, employing spectroscopic redshifts to measure the three-dimensional correlation function of X-ray AGNs. The majority of the X-ray

\footnotetext{
${ }^{14}$ Mailing address: P.O. Box 439027, San Ysidro, CA, 92143-9024, USA.
}

surveys agree with a picture where X-ray AGNs are typically hosted in DM halos with mass of the order of $12.5<$ $\log M_{\mathrm{DM}}\left(h^{-1} M_{\odot}\right)<13.5$, at low $(z<0.4)$ and high $(z \sim 1)$ redshift (Gilli et al. 2005; Yang et al. 2006; Gilli et al. 2009; Hickox et al. 2009; Coil et al. 2009; Krumpe et al. 2010; Cappelluti et al. 2010). This implies that X-ray AGNs more likely reside in massive DM halos and preferentially inhabit a dense environment typical of galaxy groups.

There have been attempts to detect the X-ray luminosity dependence of the clustering. At $z \sim 1$, neither Gilli et al. (2009) nor Coil et al. (2009) found significant dependence of the clustering amplitudes on the optical luminosity, X-ray luminosity, or hardness ratio, partially due to the larger statistical errors. Recent works by Krumpe et al. (2010) and Cappelluti et al. (2010) found, however, that high X-ray luminosity AGNs cluster more strongly than low X-ray luminosity ones at $2 \sigma$ level for $z \sim 0.3$ and $z \sim 0$, respectively.

Until recently, the clustering of AGNs has been studied mainly in the optical, particularly in large area surveys such 
as the 2dF (2QZ; Croom et al. 2005; Porciani \& Norberg 2006) and the Sloan Digital Sky Survey (SDSS; Li et al. 2006; Shen et al. 2009; Ross et al. 2009). Croom et al. (2005) analyzed the clustering of 2QZ QSOs as a function of redshift finding a strong evolution of QSO bias, with $b_{Q}(z=0.53)=1.13 \pm 0.18$ at low redshift and $b_{Q}(z=2.48)=4.24 \pm 0.53$ at high redshift, as also observed in Porciani \& Norberg (2006). The evidence of an evolution over time of the bias factor for SDSS quasars has been found in Shen et al. (2009), with bias values ranging from $b_{Q}(z=0.50)=1.32 \pm 0.17$ to $b_{Q}(z=3.17)=7.76 \pm 1.44$. The results from these surveys have also shown that the bias evolution of optically selected quasars is consistent with an approximately constant mass at all redshifts of the hosting DM halo in the range $\log M_{\mathrm{DM}} \sim 12.5-13\left(h^{-1} M_{\odot}\right)$.

Models of major mergers between gas-rich galaxies appear to naturally produce the bias of quasars as a function of $L$ and z (Hopkins et al. 2008; Shen 2009; Shankar et al. 2009, 2010; Shankar 2010; Bonoli et al. 2009), supporting the observations that bright quasars host galaxies presenting a preference for merging systems. It is still to be verified if the results from optical surveys can be extended to the whole AGN population and in particular to the X-ray selected AGNs.

In this paper, we concentrate on the study of the bias evolution with redshift using different X-ray AGN samples and we focus on the estimation of the bias factor and the hosting halo mass using a new method which properly accounts for the sample variance and the strong evolution of the bias with the time.

The paper is organized as follows. In Section 2 we describe the $X M M$ - Cosmic Evolution Survey (COSMOS) AGN sample and the AGN subsets used to estimate the correlation function. In Section 3 we describe the random catalog generated to reproduce the properties of the data sample and the method to measure the two-point statistics is explained in Section 4. The results of the AGN auto-correlation based on the standard method of the power-law fitting of the signal and using the two-halo term are given in Section 5. In Section 6 we present our own method for estimating the AGN bias factor and the DM halo masses in which AGNs reside, solving the sample variance and the bias evolution with redshift, and in Section 7 we present the results. In Section 8 we present the redshift evolution of the bias factor and the corresponding DM halo masses for the different AGN subsets. We discuss the results in the context of previous studies in Section 9 and we conclude in Section 10. Throughout the paper, all distances are measured in comoving coordinates and are given in units of Mpc $h^{-1}$, where $h=H_{0} / 100 \mathrm{~km} \mathrm{~s}^{-1}$. We use a $\Lambda$ CDM cosmology with $\Omega_{M}=0.3, \Omega_{\Lambda}=0.7$, $\Omega_{b}=0.045, \sigma_{8}=0.8$. The symbol log signifies a base-10 logarithm.

\section{AGN CATALOG}

COSMOS is a multiwavelength observational project over $1.4 \times 1.4 \mathrm{deg}^{2}$ of equatorial field centered at (R.A., decl. $)_{J 2000}=$ (150.1083, 2.210), aimed to study AGNs, galaxies, large-scale structures (LSSs) of the universe, and their co-evolution. The survey uses multiwavelength imaging from X-ray to radio bands, including the Hubble Space Telescope (Scoville et al. 2007), Subaru (Taniguchi et al. 2007), Spitzer (Sanders et al. 2007), and the Galaxy Evolution Explorer (Zamojski et al. 2007). The central $0.9 \mathrm{deg}^{2}$ of the COSMOS field has been observed in X-ray with Chandra for a total of $1.8 \mathrm{Ms}$ (Elvis et al. 2009). In addiction spectroscopic campaigns have been carried out with VIMOS/Very Large Telescope and extensive spectroscopic follow-up has been granted with the IMACS/ Magellan, MMT, and DEIMOS/KeckII projects.

XMM-Newton surveyed $2.13 \mathrm{deg}^{2}$ of the sky in the COSMOS field in the $0.5-10 \mathrm{keV}$ energy band for a total of $\sim 1.55 \mathrm{Ms}$ (Hasinger et al. 2007; Cappelluti et al. 2007, 2009) providing an unprecedented large sample of point-like X-ray sources (1822).

The XMM-COSMOS catalog has been cross-correlated with the optical multiband catalog (Cappelluti et al. 2007), the $K$-band catalog (McCracken et al. 2010), the IRAC catalog (Sanders et al. 2007; Ilbert et al. 2009), and the MIPS catalog (Le Floc'h et al. 2009). Brusa et al. (2010) presented the XMM-COSMOS multiwavelength catalog of 1797 X-ray sources with optical/ near-infrared identification, multiwavelength properties, and redshift information (from Lilly et al. 2007, 2009; Trump et al. 2007; Adelman-McCarthy et al. 2006; Prescott et al. 2006; Salvato et al. 2009).

In this paper we focus on the clustering analysis of 1465 $X M M$-COSMOS AGNs detected in the energy band 0.5-2 keV, for which we have a spectroscopic completeness of $\sim 53 \%$ (780/1465). From this sample of 780 objects we selected 593 sources with $I_{\mathrm{AB}}<23$ (this magnitude cut increases the spectroscopic completeness to about $65 \%$ ) and redshift $z<4$. The redshift distribution of the AGN sample (Figure 1, left panel) shows prominent peaks at various redshifts, $z \sim 0.12$, $z \sim 0.36, z \sim 0.73, z \sim 0.95, z \sim 1.2, z \sim 2.1$. In particular, the structure at $z \sim 0.36$ was also observed at other wavelengths in COSMOS (Lilly et al. 2007) and already discussed (Gilli et al. 2009). The median redshift of the sample is $\langle z\rangle=1.22$.

The sources have been classified as broad optical line AGNs (BL AGN, 354) and non-broad optical line AGNs (NL AGN, 239) using a combination of X-ray and optical criteria (see Brusa et al. 2010), motivated by the fact that both obscured and unobscured AGNs can be misclassified in spectroscopic studies, given that the host galaxy light may overshine the nuclear emission. Figure 2 shows the redshift distribution of BL AGNs with $\langle z\rangle=1.55$ and NL AGNs with $\langle z\rangle=0.74$.

We also studied the clustering properties of X-ray unobscured and obscured AGNs derived on the basis of the observed X-ray hardness ratio and corrected to take into account the redshift effects. In particular we used the hard X-ray band (2-10 keV) (which allows us to sample the obscured AGN population) to select a subset of $184 \mathrm{X}$-ray unobscured sources (X-unobs hereafter) with $\log N_{H}<22 \mathrm{~cm}^{-2}$ and $218 \mathrm{X}$-ray obscured (X-obs hereafter) sources with $\log N_{H} \geqslant 22 \mathrm{~cm}^{-2}$. The median redshift of the two sub-samples are $\langle z\rangle=1.12$ and $\langle z\rangle=1.30$, respectively (see Figure 2, right panel). The 47\% (40\%) of BL (NL) AGNs also have been observed in the hard band and classified as X-unobs (X-obs) AGNs.

\section{RANDOM CATALOG}

The measurements of the two-point correlation function require the construction of a random catalog with the same selection criteria and observational effects as the data to serve as an unclustered distribution to which to compare. XMM-Newton observations have varying sensitivity over the COSMOS field. In order to create an $\mathrm{AGN}$ random sample, which takes the inhomogeneity of the sensitivity over the field into account, each simulated source is placed at a random position in the sky, with flux randomly extracted from the catalog of real source fluxes (we verified that such flux selection produces the same results as if extracting the simulated sources from a reference input $\log \mathrm{N}-\log \mathrm{S})$. The simulated source is kept in the random sample if its flux is above the sensitivity map value at that 

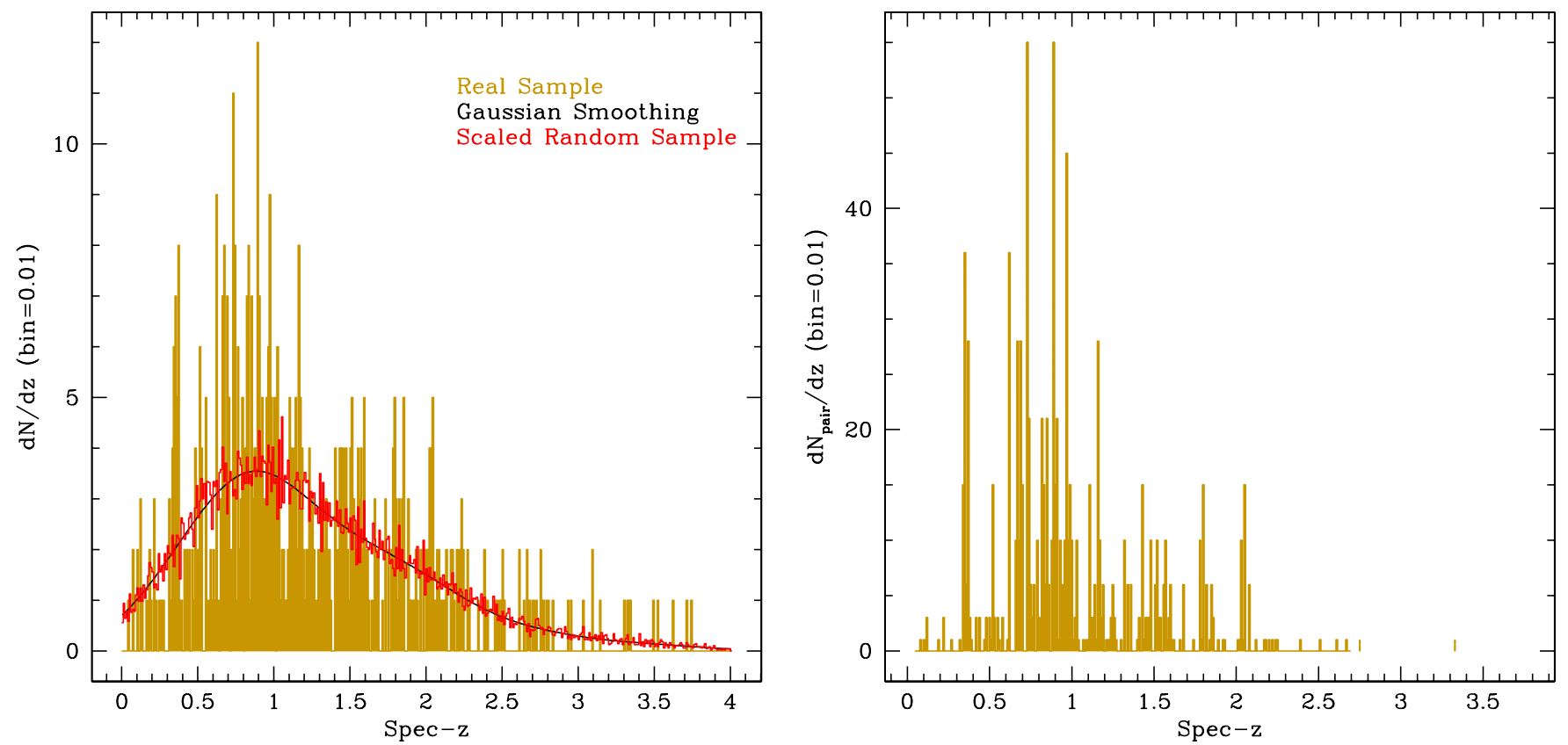

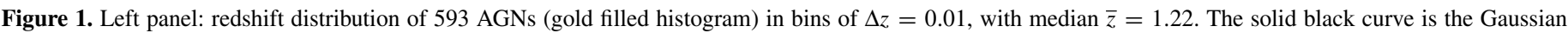

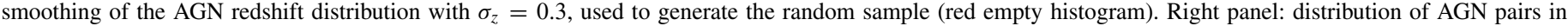
redshift bins $\Delta z=0.01$.

(A color version of this figure is available in the online journal.)
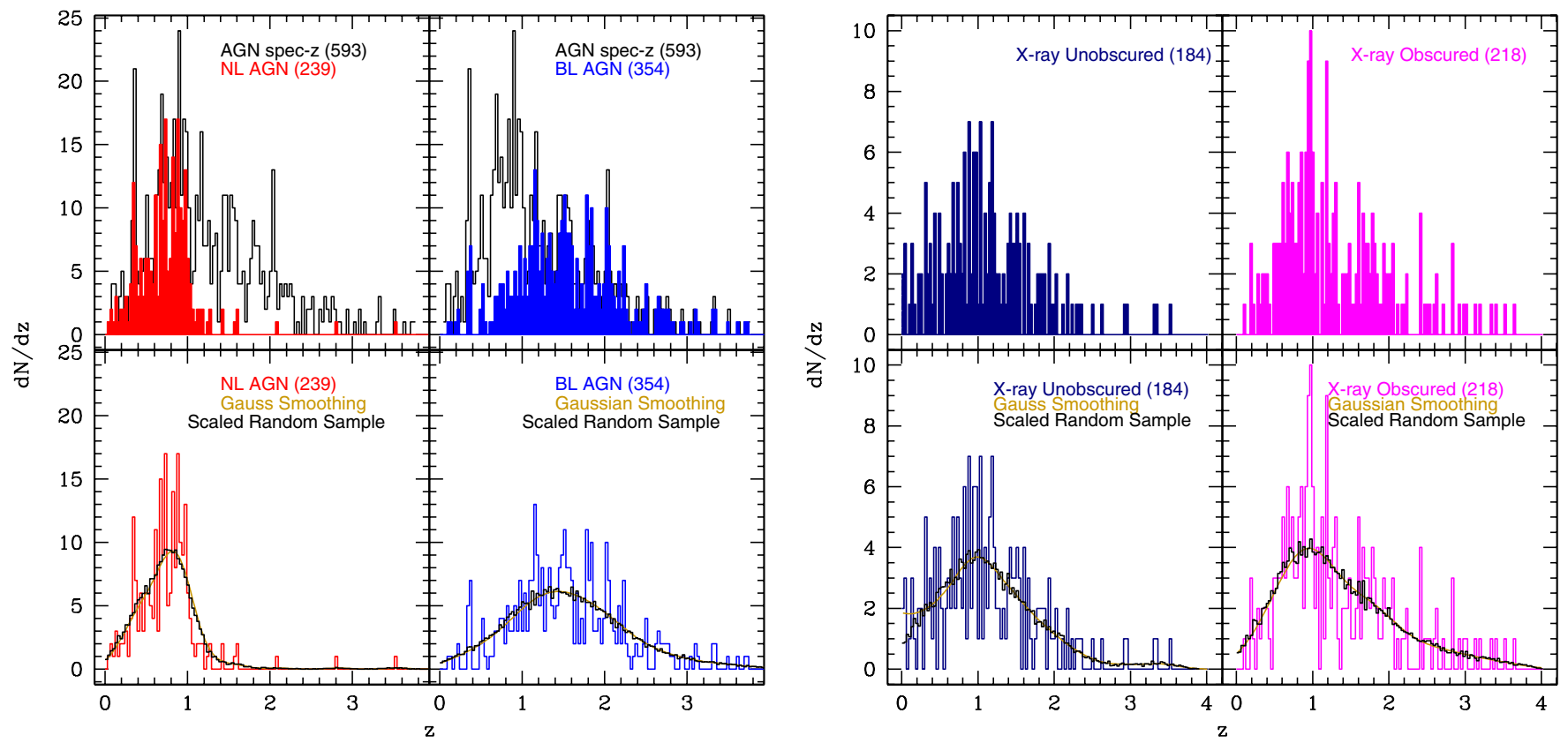

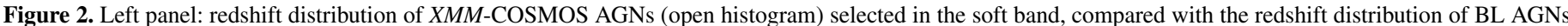

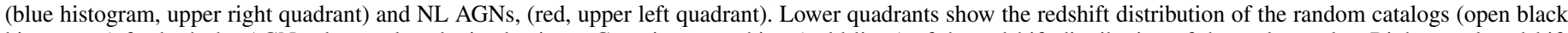

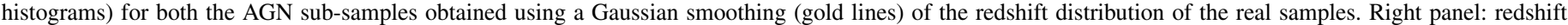

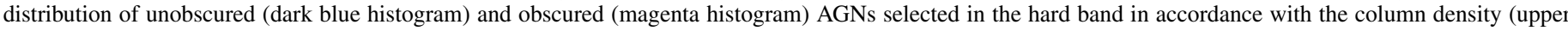

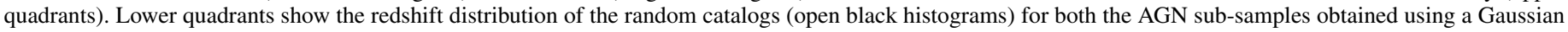
smoothing (gold lines) of the redshift distribution of the real samples.

(A color version of this figure is available in the online journal.)

position (Miyaji et al. 2007; Cappelluti et al. 2009). Placing these sources at random positions in the XMM-COSMOS field has the advantage of not removing the contribution to the signal due to angular clustering. On the other hand, this procedure does not take into account possible positional biases related to the optical follow-up program. Gilli et al. (2009), who instead decided to extract the coordinates of the random sources from the coordinate ensemble of the read sample, showed that there is a difference of only $15 \%$ in the correlation lengths measured with the two procedures.

The corresponding redshift for a random object is assigned based on the smoothed redshift distribution of the AGN sample. As in Gilli et al. (2009) we assumed a Gaussian smoothing length of $\sigma_{z}=0.3$. This is a good compromise between scales that are 
either too small, thus affected by local density variations, or too large, thus oversmoothing the distribution (our results do not change significantly using $\sigma_{z}=0.2-0.4$ ). Figure 1 (left panel) shows the redshift distribution of 593 XMM-COSMOS AGNs and the scaled random sample ( $\sim 1,000$ random sources) which follows the red solid curve obtained by Gaussian smoothing.

\section{TWO-POINT STATISTICS}

A commonly used technique for measuring the spatial clustering of a class of objects is the two-point correlation function $\xi(r)$, which measures the excess probability $d P$ above a random distribution of finding an object in a volume element $d V$ at a distance $r$ from another randomly chosen object (Peebles 1980)

$$
d P=n[1+\xi(r)] d V,
$$

where $n$ is the mean number density of objects. In particular, the auto-correlation function (ACF) measures the excess probability of finding two objects from the same sample in a given volume element. With a redshift survey, we cannot directly measure $\xi(r)$ in physical space because peculiar motions of galaxies distort the line-of-sight distances inferred from redshift. To separate the effects of redshift distortions, the spatial correlation function is measured in two dimensions, $r_{p}$ and $\pi$, where $r_{p}$ and $\pi$ are the projected comoving separations between the considered objects in the directions perpendicular and parallel, respectively, to the mean line-of-sight between the two sources. Following Davis \& Peebles (1983), $r_{1}$ and $r_{2}$ are the redshift positions of a pair of objects, $s$ is the redshiftspace separation $\left(r_{1}-r_{2}\right)$, and $l=\frac{1}{2}\left(r_{1}+r_{2}\right)$ is the mean distance to the pair. The separations between the two considered objects across $r_{p}$ and $\pi$ are defined as

$$
\begin{gathered}
\pi=\frac{\mathbf{s} \cdot \mathbf{l}}{|\mathbf{l}|} \\
r_{p}=\sqrt{\left(\mathbf{s} \cdot \mathbf{s}-\pi^{2}\right)} .
\end{gathered}
$$

Redshift-space distortions only affect the correlation function along the line of sight, so we estimate the so-called projected correlation function $w_{p}\left(r_{p}\right)$ (Davis \& Peebles 1983)

$$
w_{p}\left(r_{p}\right)=2 \int_{0}^{\pi_{\max }} \xi\left(r_{p}, \pi\right) d \pi
$$

where $\xi\left(r_{p}, \pi\right)$ is the two-point correlation function in terms of $r_{p}$ and $\pi$, measured using the Landy \& Szalay (1993; LS) estimator:

$$
\xi=\frac{1}{\mathrm{RR}^{\prime}}\left[\mathrm{DD}^{\prime}-2 \mathrm{DR}^{\prime}+\mathrm{RR}^{\prime}\right]
$$

$\mathrm{DD}^{\prime}, \mathrm{DR}^{\prime}$, and $\mathrm{RR}^{\prime}$ are the normalized data-data, data-random, and random-random number of pairs defined by

$$
\begin{aligned}
\mathrm{DD}^{\prime} & =\frac{\mathrm{DD}\left(r_{p}, \pi\right)}{n_{d}\left(n_{d}-1\right)} \\
\mathrm{DR}^{\prime} & =\frac{\mathrm{DR}\left(r_{p}, \pi\right)}{n_{d} n_{r}} \\
\mathrm{RR}^{\prime} & =\frac{\mathrm{RR}\left(r_{p}, \pi\right)}{n_{r}\left(n_{r}-1\right)},
\end{aligned}
$$

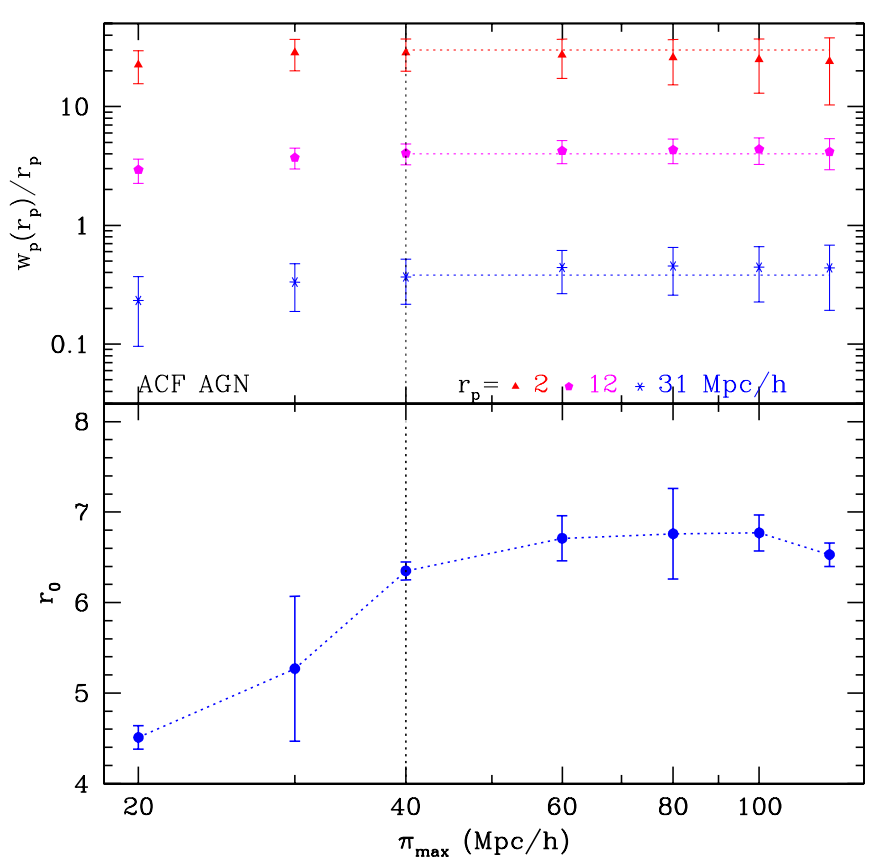

Figure 3. Projected AGN correlation function $w_{p}\left(r_{p}\right)$ computed at different $r_{p}$ scale (see label) as a function of the integral radius $\pi_{\max }$. Horizontal lines show that the ACF saturates for $\pi_{\max }>40 \mathrm{Mpc} h^{-1}$, which is also the minimum $\pi_{\max }$ at which $w_{p}\left(r_{p}\right)$ converges and returns the smaller error on the best-fit correlation parameter $r_{0}$, with $\gamma$ fixed at 1.8 .

(A color version of this figure is available in the online journal.)

where DD, DR, and RR are the number of data-data, data-random, and random-random pairs at separation $r_{p} \pm \Delta r_{p}$, and $\pi \pm \Delta \pi$ and $n_{d}, n_{r}$ are the total number of sources in the data and random sample, respectively. Figure 1 (right panel) shows the number of pairs in redshift bins $\Delta z=0.01$ for the AGN sample.

The LS estimator has been used to measure correlations in a number of surveys, for example, SDSS (Zehavi et al. 2005; Li et al. 2006), DEEP2 (Coil et al. 2007, 2008), AGES (Hickox et al. 2009), and COSMOS (Gilli et al. 2009). If $\pi_{\max }=\infty$, then we average over all line-of-sight peculiar velocities, and $w_{p}\left(r_{p}\right)$ can be directly related to $\xi(r)$ for a power-law parameterization, by

$$
w_{p}\left(r_{p}\right)=r_{p}\left(\frac{r_{0}}{r_{p}}\right)^{\gamma} \frac{\Gamma(1 / 2) \Gamma[(\gamma-1) / 2]}{\Gamma(\gamma / 2)} .
$$

In practice, we truncate the integral at a finite $\pi_{\max }$ value to maximize the correlation signal. One should avoid values of $\pi_{\max }$ that are too large since they would add noise to the estimate of $w_{p}\left(r_{p}\right)$; if instead, $\pi_{\max }$ is too small one would not recover all the signal. To determine the appropriate $\pi_{\max }$ values for the XMM-COSMOS AGN correlation function, we estimated $w_{p}\left(r_{p}\right)$ for different values of $\pi_{\max }$ in the range $20-120 \mathrm{Mpc}$ $h^{-1}$. Besides, we determined the correlation length $r_{0}$ for this set of $\pi_{\max }$ values by fitting $w_{p}\left(r_{p}\right)$ with a fixed $\gamma=1.8$ over $r_{p}$ in the range $0.5-40 \mathrm{Mpc} h^{-1}$. In Figure 3 we show the increase of the projected AGN auto-correlation $w_{p}\left(r_{p}\right)$ as a function of the integration radius $\pi_{\max }$. The $w_{p}\left(r_{p}\right)$ values appear to converge for $\pi_{\max }>40 \mathrm{Mpc} h^{-1}$. Therefore we adopt $\pi_{\max }=$ $40 \mathrm{Mpc} h^{-1}$ in the following analysis, which is the minimum $\pi_{\max }$ at which the correlation function converges. Such $\pi_{\max }$ selection returns the smallest error on the best-fit correlation parameter $r_{0}$. 

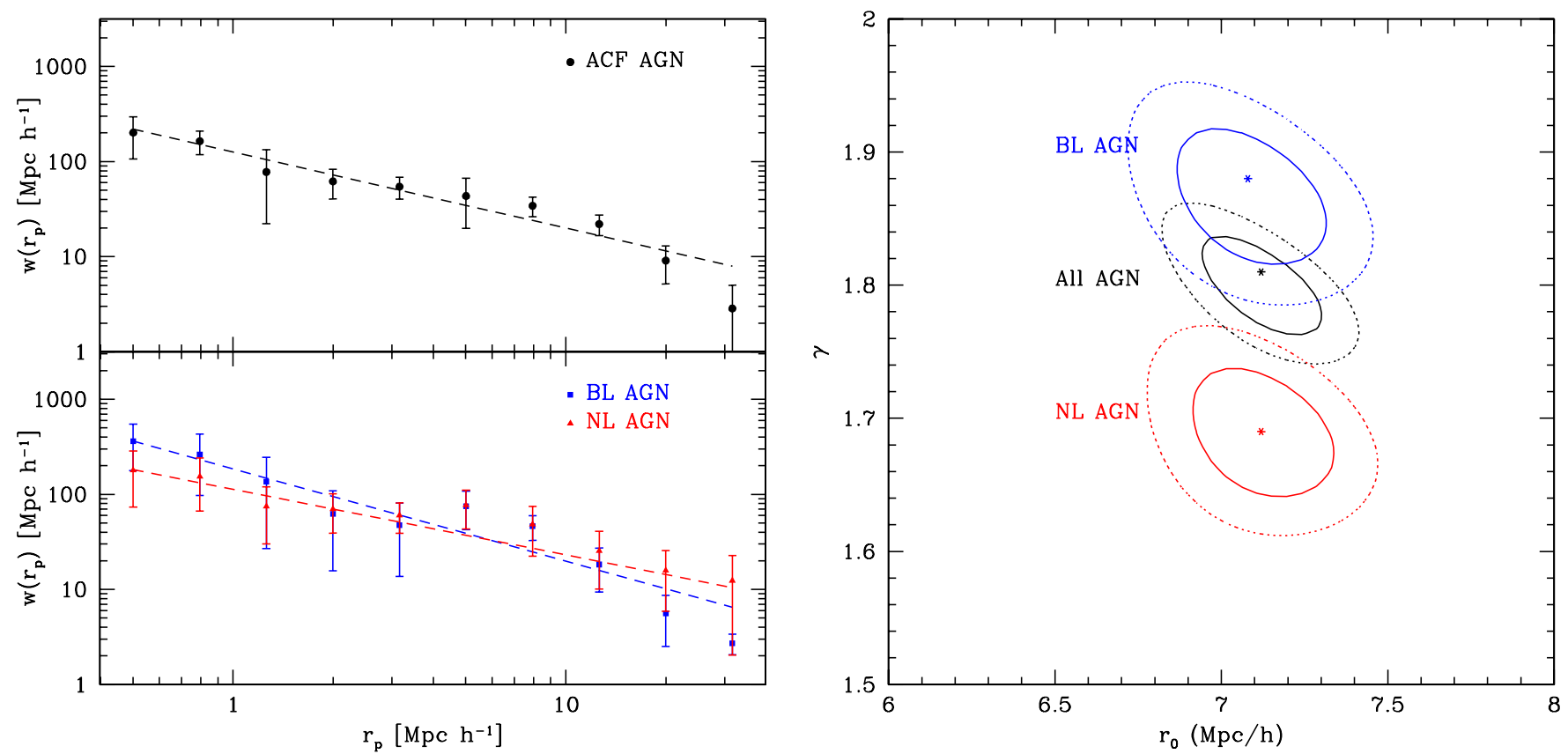

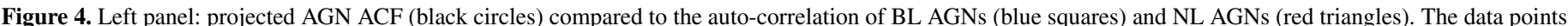

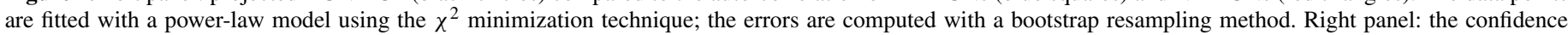

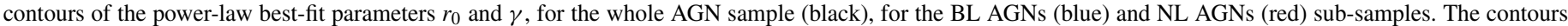
mark the $68.3 \%$ and $95.4 \%$ confidence levels (respectively corresponding to $\Delta \chi^{2}=2.3$ and 6.17) are plotted as continuous and dotted lines.

(A color version of this figure is available in the online journal.)

\section{PROJECTED AUTO-CORRELATION FUNCTION}

\subsection{Standard Approach}

To estimate the AGN ACF $\xi\left(r_{p}, \pi\right)$ using the LS formula (Equation (5)), we created a grid with $r_{p}$ and $\pi$ in the range $0.1-100 \mathrm{Mpc} h^{-1}$, in logarithmic bins $\Delta \log \left(r_{p}, \pi\right)=0.2$ and we projected $\xi\left(r_{p}, \pi\right)$ on $r_{p}$ using Equation (4).

In literature, several methods are adopted for error estimates in two-point statistics and none has been proven to be the most precise. It is known that Poisson estimators generally underestimate the variance because they do not account for the fact that the points are not statistically independent, i.e., the same objects appear in more than one pair. In this work we computed the errors on $w_{p}\left(r_{p}\right)$ with a bootstrap resampling technique (Coil et al. 2009; Hickox et al. 2009; Krumpe et al. 2010; Cappelluti et al. 2010).

The standard approach used to evaluate the power of the clustering signal is to fit $w_{p}\left(r_{p}\right)$ with a power-law model (Coil et al. 2009; Hickox et al. 2009; Gilli et al. 2009; Krumpe et al. 2010; Cappelluti et al. 2010) of the form given in Equation (9), using a $\chi^{2}$ minimization technique, with $\gamma$ and $r_{0}$ as free parameters. Figure 4 (left panel, upper quadrant) shows the projected AGN ACF, evaluated in the projected separation range $r_{p}=0.5-40 \mathrm{Mpc} h^{-1}$. The best-fit correlation length and slope and the corresponding $1 \sigma$ errors are found to be $r_{0}=7.12_{-0.18}^{+0.28} \mathrm{Mpc}^{-1}$ and $\gamma=1.81_{-0.03}^{+0.04}$.

We estimated the projected correlation function of BL and NL AGNs in the range $r_{p}=0.5-40 \mathrm{Mpc} h^{-1}$, as shown in Figure 4 (left panel, lower quadrant). For BL AGNs we found a correlation length of $r_{0}=7.08_{-0.28}^{+0.30} \mathrm{Mpc} h^{-1}$ and $\gamma=1.88_{-0.06}^{+0.04}$, while for NL AGNs we measured $r_{0}=7.12_{-0.20}^{+0.22} \mathrm{Mpc} h^{-1}$ and a flatter slope of $\gamma=1.69_{-0.05}^{+0.05}$. Figure 4 (right panel) shows the power-law best-fit parameters for the different AGN samples with the $1 \sigma$ and $2 \sigma$ confidence intervals for a two-parameter fit, which correspond to $\chi^{2}=\chi_{\min }^{2}+2.3$ and $\chi^{2}=\chi_{\min }^{2}+6.17$.

We can estimate the AGN bias factor using the power-law best-fit parameters:

$$
b_{\mathrm{PL}}=\sigma_{8, \mathrm{AGN}}(z) / \sigma_{\mathrm{DM}}(z),
$$

where $\sigma_{8, \mathrm{AGN}}(z)$ is the rms fluctuation of the density distribution over the sphere with a comoving radius of $8 \mathrm{Mpc} h^{-1}$, and $\sigma_{\mathrm{DM}}(z)$ is the DM correlation function evaluated at $8 \mathrm{Mpc} h^{-1}$, normalized to a value of $\sigma_{\mathrm{DM}}(z=0)=0.8$. For a power-law correlation function this value can be calculated by (Peebles 1980)

$$
\left(\sigma_{8, \mathrm{AGN}}\right)^{2}=J_{2}(\gamma)\left(\frac{r_{0}}{8 \mathrm{Mpc} h^{-1}}\right)^{\gamma},
$$

where $J_{2}(\gamma)=72 /\left[(3-\gamma)(4-\gamma)(6-\gamma) 2^{\gamma}\right]$. As the linear regime of the structure formation is verified only at large scales, the best-fit parameters, $r_{0}$ and $\gamma$, are estimated fitting the projected correlation function on $r_{p}=1-40 \mathrm{Mpc} h^{-1}$. The $1 \sigma$ uncertainty of $\sigma_{8, \mathrm{AGN}}$ is computed from the $r_{0}$ versus $\gamma$ confidence contour of the two-parameter fit corresponding to $\chi^{2}=\chi_{\min }^{2}+2.3$.

\subsection{Two-halo Term}

In the halo model approach, the two-point correlation function of AGNs is the sum of two contributions: the first term (onehalo term) is due to the correlation between objects in the same halo and the second term (two-halo term) arises because of the correlation between two distinct halos,

$$
w_{\mathrm{AGN}}\left(r_{p}\right)=w_{\mathrm{AGN}}^{1-h}\left(r_{p}\right)+w_{\mathrm{AGN}}^{2-h}\left(r_{p}\right) .
$$

As the two-halo term dominates at large scales, we can consider this term to be in the regime of linear density fluctuations. In the 
Table 1

Bias Factors and Hosting DM Halo Masses

\begin{tabular}{|c|c|c|c|c|}
\hline $\begin{array}{l}\text { AGN } \\
\text { Sample } \\
\text { (1) }\end{array}$ & $\langle z\rangle^{\mathrm{a}}$ & $\begin{array}{c}b_{\mathrm{PL}} \\
\text { Equation (10) } \\
\text { (3) }\end{array}$ & $\begin{array}{c}b_{2-h} \\
\text { Equation (16) } \\
\text { (4) }\end{array}$ & $\begin{array}{c}\log \bar{M}_{\mathrm{DM}}^{\mathrm{b}} \\
\left(h^{-1} M_{\odot}\right) \\
(5)\end{array}$ \\
\hline Total (593) & 1.22 & $2.80_{-0.90}^{+0.22}$ & $2.98 \pm 0.13$ & $13.23 \pm 0.06$ \\
\hline BL (354) & 1.55 & $3.11_{-1.22}^{+0.30}$ & $3.43 \pm 0.17$ & $13.14 \pm 0.07$ \\
\hline NL (239) & 0.74 & $2.78_{-1.07}^{+0.45}$ & $2.70 \pm 0.22$ & $13.54 \pm 0.10$ \\
\hline X-unobs (184) & 1.12 & $2.98_{-0.37}^{+0.34}$ & $3.01 \pm 0.21$ & $13.33 \pm 0.08$ \\
\hline X-obs (218) & 1.30 & $1.66_{-0.32}^{+0.31}$ & $1.80 \pm 0.15$ & $12.30 \pm 0.15$ \\
\hline \multicolumn{5}{|c|}{ Sub-sample at $z<1$} \\
\hline $\mathrm{BL}(70)$ & 0.57 & $2.18_{-1.02}^{+0.95}$ & $2.32 \pm 0.26$ & $13.50 \pm 0.11$ \\
\hline NL (137) & 0.53 & $1.68_{-0.57}^{+0.45}$ & $1.40 \pm 0.15$ & $12.65 \pm 0.18$ \\
\hline
\end{tabular}

Notes.

a Median redshift of the sample.

b Typical DM halo masses based on Sheth et al. (2001) and van den Bosch (2002).

linear regime, AGNs are biased tracers of the DM distribution and the AGN bias factor defines the relation between the twohalo term of DM and AGN:

$$
w_{\mathrm{AGN}}^{2-h}\left(r_{p}\right)=b_{\mathrm{AGN}}^{2} w_{\mathrm{DM}}^{2-h}\left(r_{p}\right) .
$$

We first estimated the DM two-halo term at the median redshift of the sample, using

$$
\xi_{\mathrm{DM}}^{2-h}(r)=\frac{1}{2 \pi^{2}} \int P^{2-h}(k) k^{2}\left[\frac{\sin (k r)}{k r}\right] d k,
$$

where $P^{2-h}(k)$ is the Fourier Transform of the linear power spectrum, assuming a power spectrum shape parameter $\Gamma=0.2$ and $h=0.7$. Following Hamana et al. (2002), we estimated $\xi_{\mathrm{DM}}^{2-h}(r)$ and then the DM projected correlation $w_{\mathrm{DM}}^{2-h}\left(r_{p}\right)$ using

$$
w_{\mathrm{DM}}^{2-h}\left(r_{p}\right)=2 \int_{r_{p}}^{\infty} \frac{\xi_{\mathrm{DM}}^{2-h}(r) r d r}{\sqrt{r^{2}-r_{p}^{2}}} .
$$

Using this term, we can estimate the AGN bias simply dividing the projected AGN correlation function at large scales $\left(r_{p}>1 \mathrm{Mpc} h^{-1}\right)$ by the DM two-halo term,

$$
b_{\mathrm{AGN}}^{2}=\left(w_{\mathrm{AGN}}\left(r_{p}\right) / w_{\mathrm{DM}}^{2-h}\left(r_{p}\right)\right)^{1 / 2},
$$

and then averaging over the scales $r_{p}=1-40 \mathrm{Mpc} h^{-1}$. Table 1, Column 4 shows the AGN bias factors using this method, compared with the ones based on the power-law fits of the ACF (Column 3) for the different AGN subsets. The two sets of bias values from the different approaches are consistent within $1 \sigma$, but the errors on $b_{\mathrm{PL}}$ are bigger consistently with the fact that the AGN ACF is not well described by a power law.

\section{SOLVING FOR SAMPLE VARIANCE USING HOD}

The standard approaches used in previous works on clustering of X-ray AGNs (Mullis et al. 2004; Yang et al. 2006; Gilli et al. 2005; Coil et al. 2009; Hickox et al. 2009; Krumpe et al. 2010; Cappelluti et al. 2010) to estimate the bias factors from the projected AGN ACF are based on the power-law fit parameters (method 1). This method assumes that the projected correlation function is well fitted by a power law and the bias factors are derived from the best-fit parameters, $r_{0}$ and $\gamma$, of the clustering signal at large scales.

Most of the authors (Hickox et al. 2009; Krumpe et al. 2010; Cappelluti et al. 2010) used an analytical expression (such as the one described in Sheth \& Tormen 1999; Sheth et al. 2001; Tinker et al. 2005) to assign a characteristic DM halo mass to the hosting halos. The incongruity of this approach is that the bias used is the average bias of a given sample at a given redshift. However, the average bias is sensitive to the entirety of the mass distribution so that distributions with different average masses, can give rise to the same average bias value.

In the halo model approach the large-scale amplitude signal is due to the correlation between objects in distinct halos and the bias parameter defines the relation between the large-scale clustering amplitude of the AGN ACF and the DM two-halo term $(\operatorname{method} 2)$.

At non-linear scales, we can fill DM halos with AGNs based on a statistical halo occupation distribution (HOD), allowing us to model the clustering of AGNs within halos. In the literature, the common model used for the AGN HOD is a three-parameter model including a step function for the HOD of central AGNs and a truncated power-law satellite HOD (introduced by Zehavi et al. 2005, for galaxies). Here we assumed that all the AGNs reside in central galaxies. This assumption is supported by Starikova et al. (2010). They found that X-ray AGNs are predominantly located in the central galaxies of the host DM halos and tend to avoid satellite galaxies, fixing the limit to the fraction of AGNs in non-central galaxies to be less than $10 \%$. The same fraction of satellite galaxies hosting AGN is suggested in Shen (2009). Shankar et al. (2010) modeled the measurements of quasar clustering derived in the SDSS (Shen et al. 2009) and they verified that the predicted bias factors and the correlation functions are not altered including subhalos as quasar hosts. A further consideration is that there is in practice no distinction between central and satellite AGNs in the two-halo term that we used to estimate the AGN bias factor.

We assumed a simple parametric form of the AGN halo occupation $N_{A}$, described by a delta function:

$$
N_{A}\left(M_{\mathrm{DM}}\right)=f_{A} \delta\left(M_{\mathrm{DM}}-M_{0}\right),
$$

where $f_{A}$ is the AGN duty cycle. It is clear that we are not considering the full HOD model, but we are assigning to all the AGNs the same average mass of the hosting halos. The motivation is that X-ray AGNs mainly reside in massive halos with a narrow distribution of the hosting halo masses. It is clear that this assumption is specific to AGNs and, e.g., is not applicable to galaxies.

The AGN HOD described by the $\delta$-function is motivated by the results of Miyaji et al. (2011) showing that the AGN HOD rapidly decreases at high halo masses. In addition Martini et al. (2009) and Silverman et al. (2009) found that AGNs preferentially reside in galaxy groups rather than in clusters.

The $\delta$-function is the simplest possible assumption in the treatment of the sample variance, which is due to the variation in the amplitude of source counts distribution. It has been shown in Faltenbacher et al. (2010) that the variation in the density field, which is responsible for the sample variance, can be replaced by the variation of the halo mass function. In terms of halo model, the bias factor as a function of the fluctuation $\Delta$ in the density 
field is expressed by

$$
b_{A}(\Delta)=\frac{\int_{M_{\mathrm{DM}}} N_{A}\left(M_{\mathrm{DM}}\right) b_{h}\left(M_{\mathrm{DM}}\right) n\left(M_{\mathrm{DM}}, \Delta\right) d M_{\mathrm{DM}}}{\int_{M_{\mathrm{DM}}} N_{A}\left(M_{\mathrm{DM}}\right) n\left(M_{\mathrm{DM}}, \Delta\right) d M_{\mathrm{DM}}},
$$

where $N_{A}$ is the AGN HOD, $b_{h}\left(M_{\mathrm{DM}}\right)$ is the halo bias, and $n\left(M_{\mathrm{DM}}, \Delta\right)$ is the halo mass function, which depends on the density field. On the other hand, the sample variance does not effect the AGN halo occupation. In V. Allevato et al. (2011, in preparation), we confirm the assumption of the constancy of the AGN HOD with the density field.

When we assume that all AGNs reside in DM halos with the same mass, Equation (18) becomes simpler:

$$
\frac{\int_{M_{\mathrm{DM}}} \delta\left(M_{\mathrm{DM}}-M_{0}\right) b_{h}\left(M_{\mathrm{DM}}\right) n\left(M_{\mathrm{DM}}, \Delta\right) d M_{\mathrm{DM}}}{\int_{M_{\mathrm{DM}}} \delta\left(M_{\mathrm{DM}}-M_{0}\right) n\left(M_{\mathrm{DM}}, \Delta\right) d M_{\mathrm{DM}}}=b\left(M_{0}\right) .
$$

The equation shows that when the AGN HOD is close to a $\delta$-function, the variations in the density field only change the AGN number density and put more weight on AGN bias at the redshift of LSS, but do not change the bias of AGNs inside the structure. Our claim differs from the results presented by Gilli et al. (2005) and Gilli et al. (2009). They found that by excluding sources located within an LSS, the correlation length and then the bias factor strongly reduces. Such bias behavior can be used to constrain more complicated shapes of the AGN HOD than a $\delta$-function type distribution.

However, even in the case of a $\delta$-function HOD, we still need to consider the two effects that are often omitted in the clustering analysis: the LSS growth and the evolution of the bias factor with $z$. Ignoring these effects alone can lead to a difference in the results reported for the different AGN samples.

The bias factor depends on the redshift as the structures grow over time, associated with our use of a large redshift interval. For the $i$ th source at redshift $z_{i}$, we considered the bias factor corresponding to a halo mass $M_{\mathrm{DM}}=M_{0}$ :

$$
b_{i}=b\left(M_{0}, z_{i}\right)
$$

where $b\left(M_{0}, z\right)$ is evaluated using van den Bosch (2002) and Sheth et al. (2001). For each AGN at redshift $z$ we estimated the factor $g(z)$ defined as the square root of the projected DM twohalo term at redshift $z$ normalized to the projected DM two-halo term evaluated at $z=0$,

$$
g(z)=\sqrt{\frac{w_{\mathrm{DM}}\left(z, r_{p}\right)}{w_{\mathrm{DM}}\left(z=0, r_{p}\right)}},
$$

averaged over the scales $r_{p}=1-40 \mathrm{Mpc} h^{-1}$. As the amplitude of the projected DM two-halo term decreases with increasing redshift, $g$ is a decreasing function of $z$ (see Figure 5), well described by the term $D_{1}(z) / D_{1}(z=0)$, where $D_{1}(z)$ is the growth function (see Equation (10) in Eisenstein \& Hu (1999), and references therein).

By accounting for the fact that the linear regime of the structure formation is verified only at large scales, we estimated the AGN bias considering only the pairs that contribute to the AGN clustering signal at $r_{p}=1-40 \mathrm{Mpc} h^{-1}$. We defined the weighted bias factor of the sample as

$$
\bar{b}\left(M_{0}\right)=\sqrt{\frac{\sum_{i, j} b_{i} b_{j} g_{i} g_{j}}{N_{\text {pair }}}},
$$

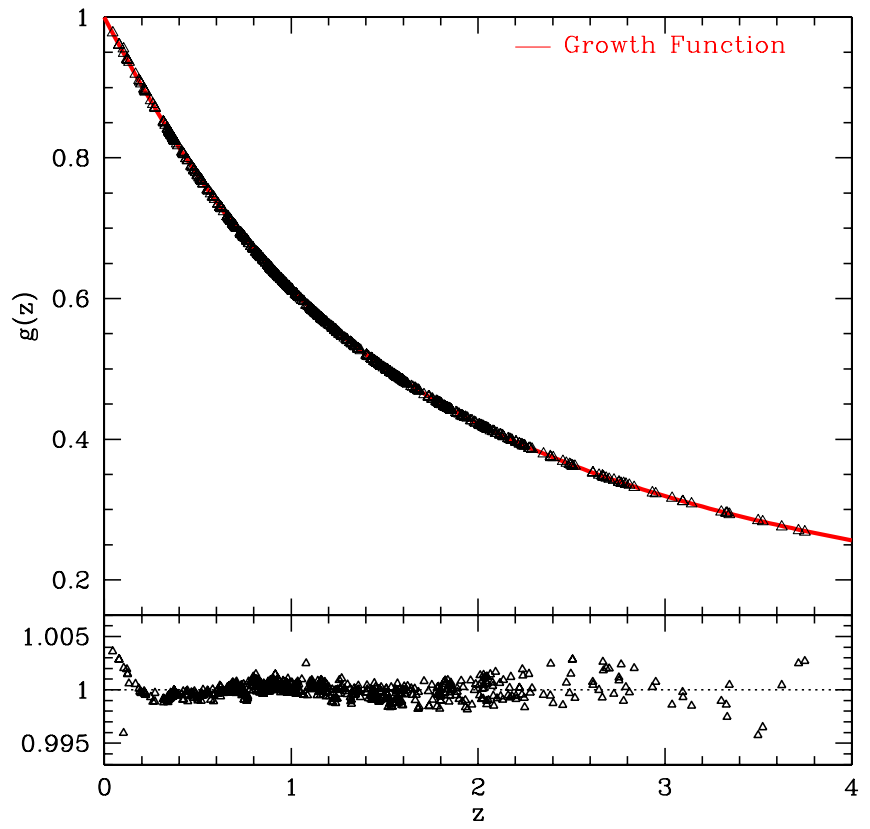

Figure 5. Factor $g$ as defined in Equation (21), estimated at the redshift of each AGN (black triangles), compared to the growth function $D_{1}(z) / D_{1}(z=0)$ (redline, see Equation (10) in Eisenstein \& Hu 1999, and references therein). The lower panel shows the ratio between the data and the model prediction. The bias of each AGN is weighted by this factor according to the redshift $z$ of the source.

(A color version of this figure is available in the online journal.)

where $b_{i} b_{j}$ is the bias factor of the $i$ th and $j$ th source in the pair $i-j, g_{i} g_{j}$ is the $g$ factor of the pair, and $N_{\text {pair }}$ is the total number of pairs in the range $r_{p}=1-40 \mathrm{Mpc} h^{-1}$.

Similarly, we defined a weighted average redshift of the AGN sample, weighting the redshift of each pair for the $g$ factor and the bias of the pair $\left(b_{i} b_{j}\right)$ :

$$
\bar{z}=\frac{\sum_{i, j} b_{i} b_{j} g_{i} g_{j} z_{\text {pair }}}{\sum_{i, j} b_{i} b_{j} g_{i} g_{j}}
$$

where $z_{\text {pair }}=\left(z_{i}+z_{j}\right) / 2$. Following this approach, we can find the value of $M_{0}$ that satisfies

$$
b_{1}=\bar{b}\left(M_{0}\right)
$$

where $b_{1}$ is the square root of the projected AGN ACF normalized to the projected DM two-halo term at $z=0$,

$$
b_{1}=\sqrt{\frac{w_{\mathrm{AGN}}\left(r_{p}\right)}{w_{\mathrm{DM}}\left(z=0, r_{p}\right)}},
$$

averaged over the scale $r_{p}=1-40 \mathrm{Mpc} h^{-1}$.

By performing the test in narrow redshift intervals, we can study the dependency of the halo mass $M_{0}$ on redshift (see Section 8). Moreover with just a single measurement of the amplitude of the two-halo term, one cannot constrain the AGN HOD. Already with several measurements sampling different density fields, the shape of the HOD can be linked to the LSS density dependence of the bias. In addition, the one-halo term of the AGN auto-correlation and AGN-groups cross-correlation can be used to discriminate between different HOD models, which will be the argument of our future work. 


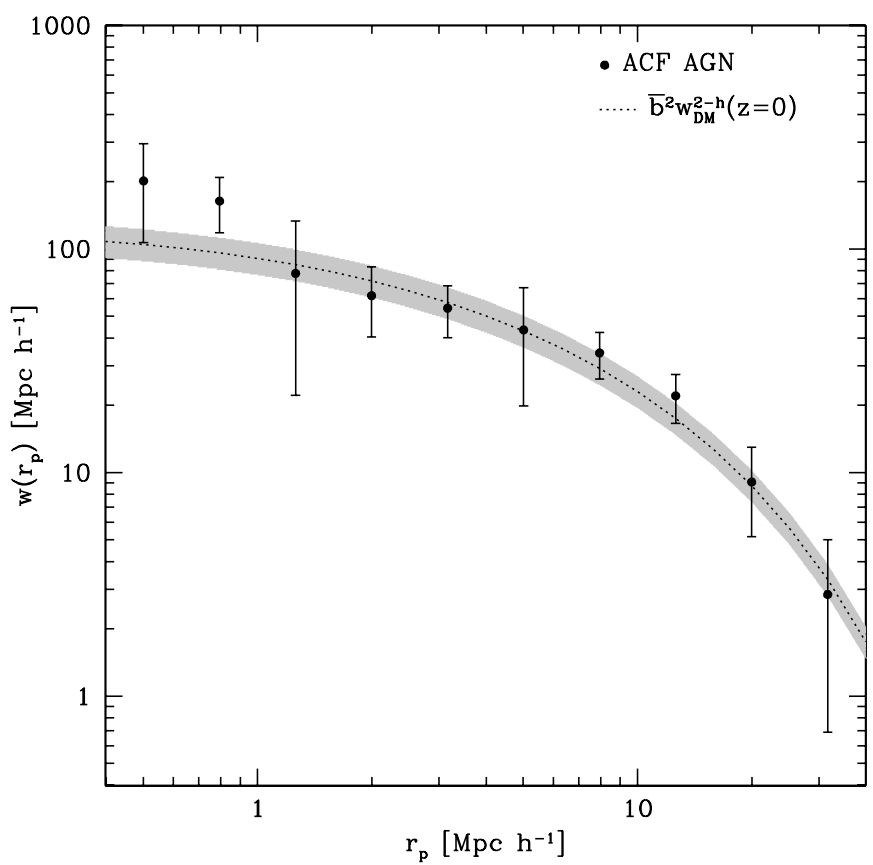

Figure 6. Projected AGN ACF (black circles) compared to $\bar{b}^{2} w_{\mathrm{DM}}^{2-h}\left(r_{p}, z=0\right)$ (dotted line), where the weighed bias $\bar{b}$ is defined in Equation (22). The shaded region shows the projected DM two-halo term scaled by $(\bar{b} \pm \delta \bar{b})^{2}$.

\section{MEASUREMENTS}

The weighted bias $\bar{b}$ and redshift $\bar{z}$, and the corresponding DM halo masses $M_{0}$ are shown in Table 2 for the different AGN sub-samples. The values have been estimated using the method described in the previous section.

Figures 6-8 show the ACF of the whole AGN, BL/NL AGN, and X-unobs/obs AGN samples compared to the term $\bar{b}^{2} w_{\mathrm{DM}}^{2-h}\left(r_{p}, z=0\right)$ (dotted line), where the weighed bias $\bar{b}$ is

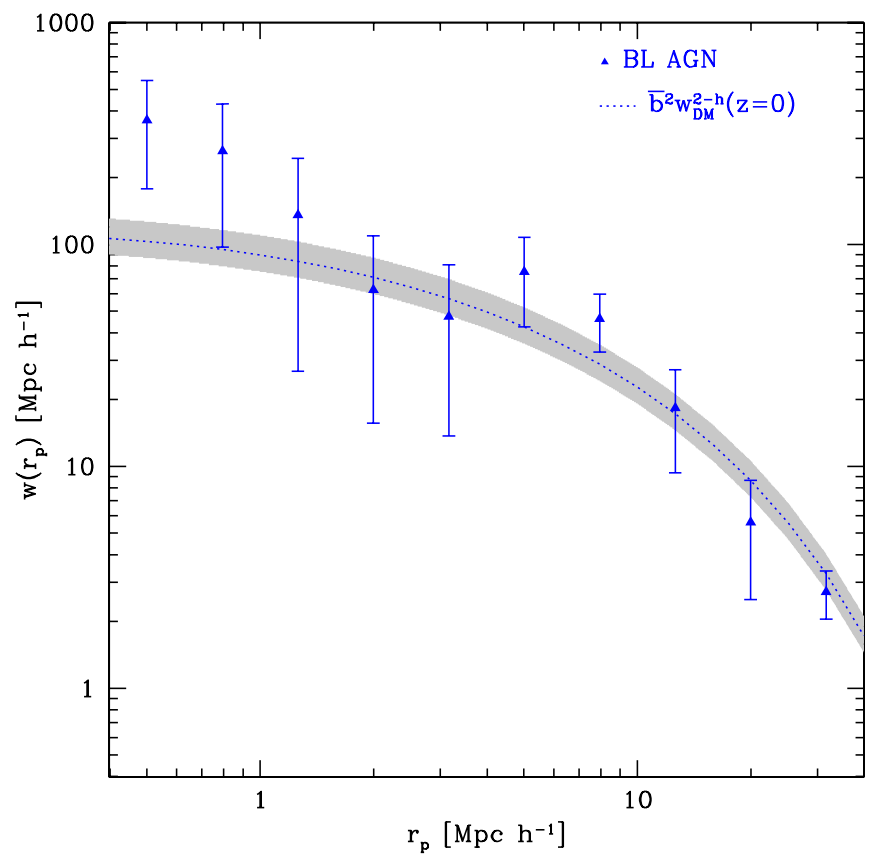

Table 2

Weighted Bias Factors and Hosting DM Halo Masses

\begin{tabular}{lcccc}
\hline \hline $\begin{array}{l}\text { AGN } \\
\text { Sample }\end{array}$ & $\begin{array}{c}\bar{b} \\
\text { Equation (22) } \\
(1)\end{array}$ & $\begin{array}{c}\bar{z} \\
\text { Equation (23) }\end{array}$ & $\begin{array}{c}\log M_{0} \\
\left(h^{-1} M_{\odot}\right)\end{array}$ & $b_{S 01}{ }^{a}$ \\
\hline Total (593) & $1.91 \pm 0.13$ & 1.21 & $13.10 \pm 0.06$ & $2.71 \pm 0.14$ \\
BL (354) & $1.74 \pm 0.17$ & 1.53 & $13.24 \pm 0.06$ & $3.68 \pm 0.27$ \\
NL (239) & $1.80 \pm 0.22$ & 0.82 & $13.01 \pm 0.08$ & $2.00 \pm 0.12$ \\
X-unobs (184) & $1.95 \pm 0.21$ & 1.16 & $13.30 \pm 0.10$ & $3.01 \pm 0.26$ \\
X-obs (218) & $1.37 \pm 0.15$ & 1.02 & $12.97 \pm 0.08$ & $2.23 \pm 0.13$ \\
Sub-sample at $z$ & 1 & & & \\
$\quad$ BL (70) & $1.62 \pm 0.26$ & 0.63 & $13.27 \pm 0.10$ & $1.95 \pm 0.17$ \\
$\quad$ NL (137) & $1.56 \pm 0.15$ & 0.60 & $12.97 \pm 0.07$ & $1.62 \pm 0.15$ \\
\hline
\end{tabular}

Note. ${ }^{\text {a }}$ Bias estimated from $M_{0}$ using Sheth et al. (2001).

defined in Equation (22). The shaded region shows the projected DM two-halo term scaled by $(\bar{b} \pm \delta \bar{b})^{2}$.

The AGN bias estimates indicate that XMM-COSMOS AGNs reside in halos with an average mass of $\log M_{0}=13.01 \pm$ $0.09\left(h^{-1} \log M_{\odot}\right)$, characteristic of moderate-size poor groups, a result consistent with previous works on X-ray selected AGNs that indicates that the typical DM halo mass hosting AGN is in the range $12.5 \lesssim \log M_{\mathrm{DM}} \lesssim 13.5\left(h^{-1} M_{\odot}\right)$

We found that BL and NL AGNs, which peak at $\bar{z}=1.53$ and $\bar{z}=0.82$, present consistent bias factors that correspond to $\mathrm{DM}$ halo average masses of $\log M_{0}=13.24 \pm 0.06\left(h^{-1} M_{\odot}\right)$ and $13.01 \pm 0.08\left(h^{-1} M_{\odot}\right)$, respectively. As described in Brusa et al. (2010), only a small fraction of the objects classified as NL AGNs are located at $z>1$, compared with 350 in the BL AGN sample. This is mostly due to the fact that highredshift NL AGNs are optically faint (typically $I \sim 23-24$ ) and have not been targeted yet with dedicated spectroscopic campaigns. Our results might be affected by the limitations in the obscured AGN classification, considering that some models on the evolution of the obscured AGN fraction predict

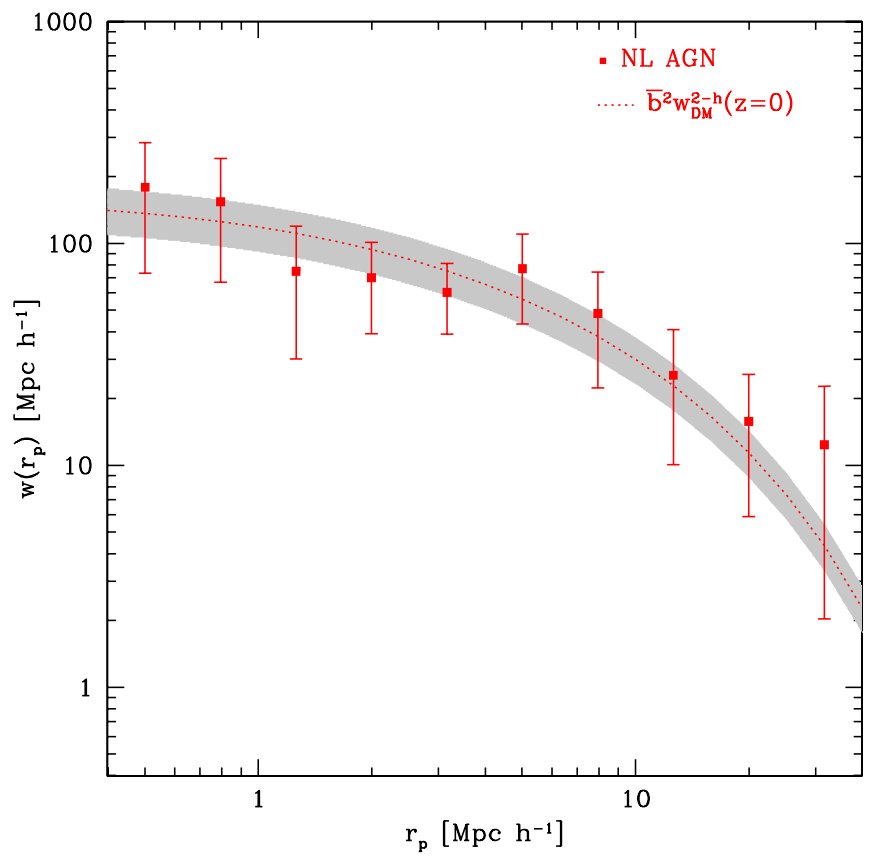

Figure 7. Projected ACF of BL AGNs (blue triangles, left panel) and NL AGNs (red squares, right panel), compared to $\bar{b}^{2} w_{\mathrm{DM}}^{2-h}\left(r_{p}, z=0\right)(\mathrm{dotted}$ line), where the weighed bias $\bar{b}$ is defined in Equation (22). The shaded region shows the projected DM two-halo term scaled by $(\bar{b} \pm \delta \bar{b})^{2}$.

(A color version of this figure is available in the online journal.) 

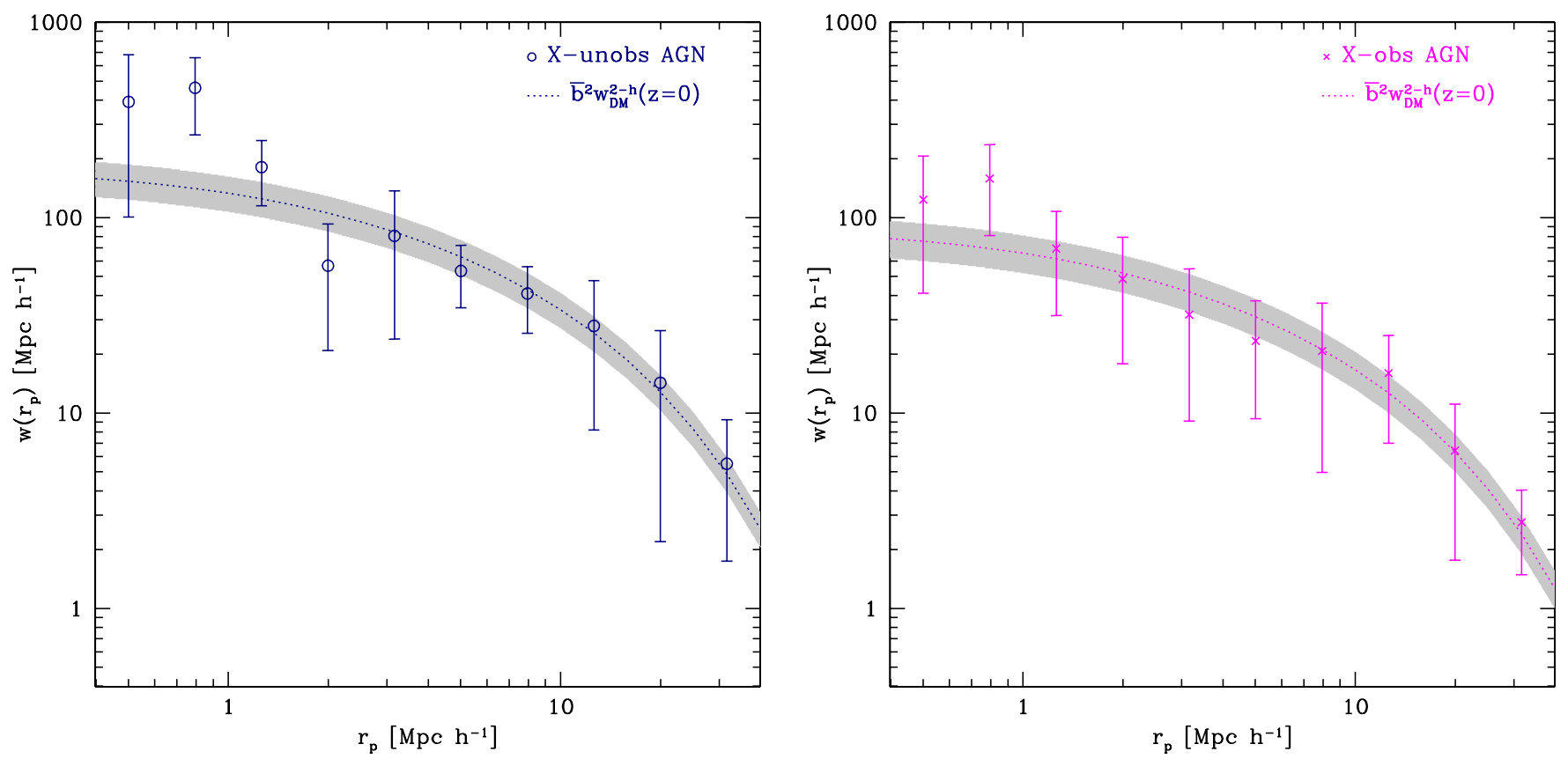

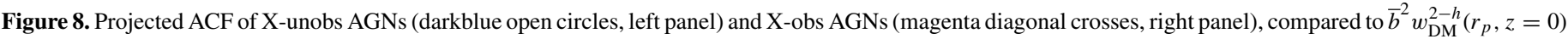
(dotted line), where the weighed bias $\bar{b}$ is defined in Equation (22). The shaded region shows the projected DM two-halo term scaled by $(\bar{b} \pm \delta \bar{b})^{2}$.

(A color version of this figure is available in the online journal.)

an increase of the fraction with redshift (Hasinger 2008). In order to avoid the problem of different redshift distribution in comparing BL/NL AGN clustering amplitude, we selected for each sample a subset (BL AGNs with 70 sources and NL AGNs with 137) at $\bar{z} \sim 0.6$. At the same redshift we found that $\mathrm{BL}$ and NL AGNs have a bias factor $\bar{b}_{\mathrm{BL}}=1.62 \pm 0.26$ and $\bar{b}_{\mathrm{NL}}=1.56 \pm 0.15$, which correspond to average halo masses of $\log M_{0}=13.27 \pm 0.10\left(h^{-1} M_{\odot}\right)$ and $12.97 \pm 0.07\left(h^{-1} M_{\odot}\right)$, respectively.

Similar results have been obtained using $\mathrm{X}$-unobs and $\mathrm{X}$-obs AGN samples; unobscured AGNs at $\bar{z}=1.16$ inhabit halos with an average mass of $\log M_{0}=13.30 \pm 0.10\left(h^{-1} M_{\odot}\right)$ which is higher at the $2.5 \sigma$ level than the halo mass hosting obscured AGNs $\left(\log M_{0}=12.97 \pm 0.08\left(h^{-1} M_{\odot}\right)\right)$ at similar redshift.

In order to compare our results with previous works on the bias of X-ray selected AGNs, we evaluated the bias factors corresponding to the halo mass $M_{0}$ at $\bar{z}$ using Sheth et al. (2001) as shown in Table 2, Column 5.

Our results support the picture that at a given redshift, $\mathrm{X}$-ray selected BL/X-unobs AGNs reside in more massive halos compared to X-ray selected NL/X-obs AGNs. This result would be expected if the two classes of AGNs correspond to different phases of the AGN evolution sequence (Hopkins et al. 2006, 2008; Hickox et al. 2009).

\section{BIAS EVOLUTION AND CONSTANT MASS THRESHOLD}

In order to investigate the redshift evolution of the bias factor, we split the $X M M$-COSMOS AGN sample into three redshift bins. The sizes of the redshift bins have been determined such that there are more or less the same number of objects in each bin. The values of $\bar{b}, \bar{z}$, and $M_{0}$ for the total AGN sample are shown in Table 3. The meaning of the table columns are (1) sample, (2) number of sources, (3) bias parameter from the projected DM two-halo term, evaluated at the median $\langle z\rangle$ of the sample, (4) typical halo mass using van den Bosch (2002) and Sheth et al. (2001), (5) weighted bias of the sample, (6) weighted redshift of the sample, (7) average DM halo mass, and (8) bias factor from $M_{0}$ estimated using Sheth et al. (2001).

We observed an increase in the AGN bias factor with redshift, from $\bar{b}(\bar{z}=0.92)=1.80 \pm 0.19$ to $\bar{b}(\bar{z}=1.94)=$ $2.63 \pm 0.21$ with a DM halo mass consistent with being constant at $\log M_{0}\left(h^{-1} M_{\odot}\right) \sim 13.1$ in each bin. These results support the picture that the bias of $X M M$-COSMOS AGNs evolves with time according to a constant halo mass track at all redshifts $z<2$.

This conclusion, based on the analysis of the global XMMCOSMOS AGN sample, can, however, be affected by the fact that the relative proportions of BL and NL AGNs are a strong function of redshift. In fact, since the XMM-COSMOS AGN sample is a flux limited sample, more luminous AGNs are selected at high redshift and, also because of our magnitude limit, high- $z$ sources in our sample are mainly BL AGNs (see Section 2). For this reason the BL AGN sample could be analyzed up to $z \sim 2.25$, while the maximum average redshift of the two redshift bins for NL AGNs is $z \sim 0.91$. We found evidence of a strong increase in the BL AGN bias factor in four redshift bins (see Table 3), with a DM halo mass constant at $\log M_{0}\left(h^{-1} M_{\odot}\right) \sim 13.28$ at all redshifts $z<2.25$. For NL AGNs we estimated $\bar{b}(\bar{z}=0.62)=1.59 \pm 0.13$ and $\bar{b}(\bar{z}=0.91)=1.87 \pm 0.19$, which correspond to a constant halo mass values $\log M_{0}\left(h^{-1} M_{\odot}\right) \sim 13.02$. We split the $\mathrm{X}$-unobs and X-obs AGN samples into two redshift bins up to $\bar{z} \simeq 1.5$ and we found that the bias of X-unobs AGNs (X-obs AGNs) evolves according to a constant halo mass consistent with the mass of BL AGNs (NL AGNs) hosting halos. Figure 9 (left panel) shows the redshift evolution of the average DM halo mass $M_{0}$ for all the AGN subsets. The horizontal lines represent the mean value of $M_{0}$ for BL/X-unobs AGNs (dashedblue), NL/X-obs AGNs (long dashed-red), and for the whole AGN sample (dotted-black). Figure 9 (right panel) shows the 

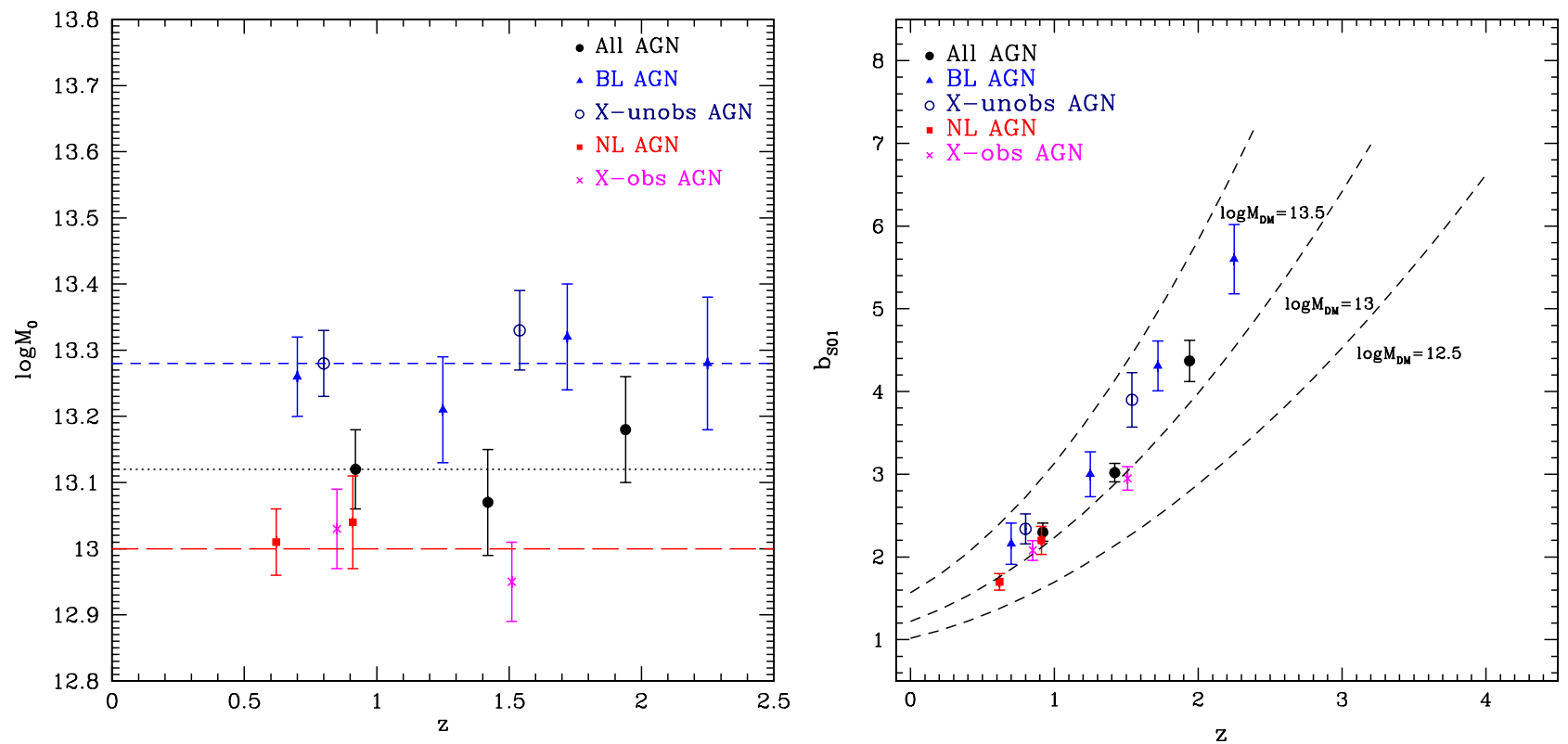

Figure 9. Left panel: DM halo mass $M_{0}$ as a function of $z$ for different AGN sub-samples (see the legend). The horizontal lines show the mean value of $M_{0}$ for BL/X-unobs AGN (dashed blue), NL/X-obs AGN (long dashed red) and for the whole AGN sample (dotted black). Right panel: redshift evolution of the bias parameter $b_{S 01}$ of different AGN sub-samples. The dashed lines show the expected $b(z)$ of typical DM halo masses $M_{\mathrm{DM}}$ based on Sheth et al. (2001). The masses are given in $\log M_{\mathrm{DM}}$ in units of $h^{-1} M_{\odot}$. BL/X-unobs AGNs present a strong bias evolution with redshift with a constant DM halo mass $\log M_{0}=13.28 \pm 0.07\left(h^{-1} M_{\odot}\right)$ up to $\bar{z} \sim 2.4$. NL/X-obs AGNs reside in less massive halos with $\log M_{0}=13.00 \pm 0.06\left(h^{-1} M_{\odot}\right)$, constant at $\bar{z}<1.5$.

(A color version of this figure is available in the online journal.)

Table 3

Bias Evolution

\begin{tabular}{|c|c|c|c|c|c|c|c|}
\hline$\overline{\langle z\rangle^{\mathrm{a}}}$ & $N$ & $\begin{array}{c}b_{2-h} \\
\text { Equation (16) } \\
\text { (3) }\end{array}$ & $\begin{array}{c}\log \bar{M}_{\mathrm{DM}}^{\mathrm{b}} \\
\left(h^{-1} M_{\odot}\right) \\
(4)\end{array}$ & (5) & $(6)$ & $\begin{array}{c}\log M_{0} \\
\text { Equation (16) } \\
(7)\end{array}$ & $\begin{array}{c}b_{S 01}{ }^{\mathrm{c}} \\
\left(h^{-1} M_{\odot}\right) \\
(8)\end{array}$ \\
\hline \multicolumn{8}{|c|}{ All AGNs } \\
\hline 0.80 & 190 & $2.70 \pm 0.19$ & $13.48 \pm 0.10$ & $1.80 \pm 0.19$ & 0.92 & $13.12 \pm 0.06$ & $2.30 \pm 0.11$ \\
\hline 1.30 & 220 & $3.10 \pm 0.18$ & $13.21 \pm 0.10$ & $2.14 \pm 0.18$ & 1.42 & $13.07 \pm 0.08$ & $3.02 \pm 0.11$ \\
\hline 2.07 & 183 & $5.18 \pm 0.21$ & $13.30 \pm 0.11$ & $2.63 \pm 0.21$ & 1.94 & $13.18 \pm 0.08$ & $4.37 \pm 0.27$ \\
\hline \multicolumn{8}{|c|}{ BL AGNs } \\
\hline 0.67 & 70 & $2.62 \pm 0.20$ & $13.57 \pm 0.10$ & $1.52 \pm 0.20$ & 0.70 & $13.26 \pm 0.06$ & $2.16 \pm 0.25$ \\
\hline 1.25 & 108 & $3.06 \pm 0.23$ & $13.24 \pm 0.08$ & $2.02 \pm 0.23$ & 1.25 & $13.21 \pm 0.08$ & $3.00 \pm 0.27$ \\
\hline 1.71 & 92 & $5.37 \pm 0.28$ & $13.60 \pm 0.08$ & $3.57 \pm 0.28$ & 1.72 & $13.32 \pm 0.08$ & $4.31 \pm 0.30$ \\
\hline 2.46 & 85 & $6.82 \pm 0.27$ & $13.41 \pm 0.10$ & $4.02 \pm 0.27$ & 2.25 & $13.28 \pm 0.10$ & $5.60 \pm 0.42$ \\
\hline \multicolumn{8}{|c|}{ X-unobscured AGNs } \\
\hline 0.65 & 98 & $2.46 \pm 0.17$ & $13.51 \pm 0.11$ & $1.62 \pm 0.17$ & 0.80 & $13.28 \pm 0.05$ & $2.34 \pm 0.18$ \\
\hline 1.66 & 86 & $4.85 \pm 0.18$ & $13.51 \pm 0.10$ & $2.10 \pm 0.18$ & 1.54 & $13.33 \pm 0.06$ & $3.90 \pm 0.33$ \\
\hline \multicolumn{8}{|c|}{ NL AGNs } \\
\hline 0.53 & 137 & $1.40 \pm 0.13$ & $12.65 \pm 0.12$ & $1.59 \pm 0.13$ & 0.62 & $13.01 \pm 0.05$ & $1.70 \pm 0.10$ \\
\hline 1.02 & 102 & $2.11 \pm 0.19$ & $12.88 \pm 0.15$ & $1.87 \pm 0.19$ & 0.91 & $13.04 \pm 0.07$ & $2.20 \pm 0.17$ \\
\hline \multicolumn{8}{|c|}{ X-obscured AGNs } \\
\hline 0.73 & 106 & $1.80 \pm 0.14$ & $13.01 \pm 0.11$ & $1.51 \pm 0.14$ & 0.85 & $13.03 \pm 0.06$ & $2.08 \pm 0.12$ \\
\hline 1.84 & 112 & $3.51 \pm 0.16$ & $12.94 \pm 0.13$ & $1.96 \pm 0.16$ & 1.51 & $12.95 \pm 0.06$ & $2.95 \pm 0.14$ \\
\hline
\end{tabular}

Notes.

a Median redshift of the sample.

b Typical DM halo masses based on Sheth et al. (2001) and van den Bosch (2002).

${ }^{\mathrm{c}}$ Bias estimated from $M_{0}$ using Sheth et al. (2001).

redshift evolution of the bias factors $b_{S 01}$ (Table 3 , Column 7) for different AGN sub-samples. The dashed lines show the expected $b(z)$ associated to the typical DM halo mass based on Sheth et al. (2001).

These results show that X-ray selected BL/X-unobs AGNs reside in more massive DM halos compared to X-ray selected $\mathrm{NL} / \mathrm{X}$-obs AGNs at all redshifts $z$ at $\sim 3 \sigma$ level. This suggests that the AGN activity is a mass triggered phenomenon and that different AGN phases are associated with the DM halo mass, irrespective of redshift $z$.

\section{DISCUSSION}

\subsection{Which DM Halos Host X-Ray AGNs?}

We have introduced a new method that uses the two-halo term in estimating the AGN bias factor and that properly 


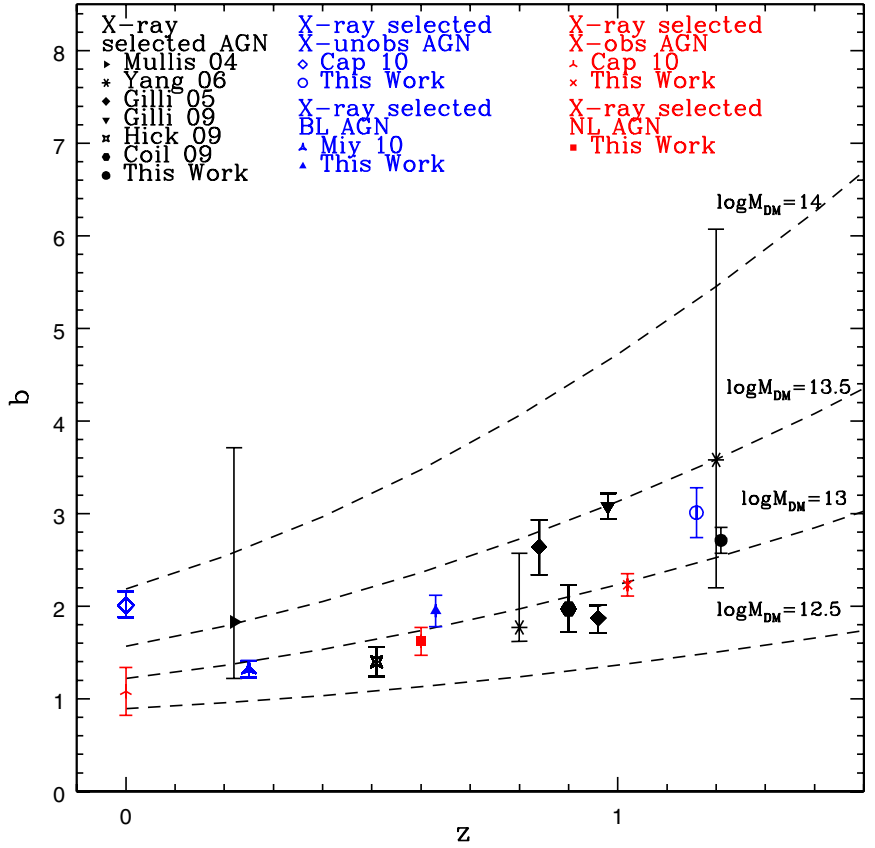

Figure 10. Bias parameter as a function of redshift for various X-ray selected AGNs (black data points), X-ray selected BL/X-unobs AGNs (blue data points), and X-ray selected NL/X-obs AGNs (red data points) as estimated in previous studies and in this work according to the legend. Our results refer to the bias factor $b_{S 01}$ showed in Table 2, Column 5. The dashed lines show the expected $b(z)$ of typical DM halo masses $M_{\mathrm{DM}}$ based on Sheth et al. (2001). The masses are given in $\log M_{\mathrm{DM}}$ in units of $h^{-1} M_{\odot}$.

(A color version of this figure is available in the online journal.)

accounts for the sample variance and the growth of the structures over time associated with our use of a large redshift interval of the AGN sample. Using this approach we have estimated an average mass of the XMM-COSMOS AGN-hosting halos equal to $\log M_{0}\left(h^{-1} \mathrm{Mpc}\right)=13.10 \pm 0.06$ which differs at $\sim 1.6 \sigma$ level from the typical halo mass $M_{\mathrm{DM}}$ based on Sheth et al. (2001) using method 2 (see Section 5.2). The difference between the standard method and our own method is also clear for the mass of BL and NL AGN-hosting halos. We have found that BL AGNs inhabit DM halos with an average mass of $\log M_{0}\left(h^{-1} \mathrm{Mpc}\right)=13.24 \pm 0.06$ at $\bar{z}=$ 1.53, while halos hosting NL AGNs have an average mass of $\log M_{0}\left(h^{-1} \mathrm{Mpc}\right)=13.01 \pm 0.08$. BL AGNs reside in more massive halos than NL AGNs also selecting two sub-samples that peak at the same median redshift $\bar{z} \sim 0.6$. We obtained similar results using X-ray unobscured AGNs at $\bar{z}=1.16$ and X-ray obscured AGNs at $\bar{z}=1.02\left(\log M_{0}\left(h^{-1} \mathrm{Mpc}\right)=13.30 \pm\right.$ 0.10 and $\log M_{0}\left(h^{-1} \mathrm{Mpc}\right)=12.97 \pm 0.08$, respectively $)$.

Instead the typical halo mass based on Sheth et al. (2001) using the AGN bias estimated with method 2 strongly depends on the median redshift of the sample. According to method 2, BL AGNs at $\langle z\rangle=1.55$ reside in less massive halos compared to NL AGNs at $\langle z\rangle=0.74$, while the result is different selecting two samples of BL and NL AGNs at the same $\langle z\rangle \sim 0.5$. Our results agree with the majority of the recent studies of $\mathrm{X}$-ray surveys which suggest a picture in which X-ray AGNs are typically hosted in DM halos with masses in the range $12.5<\log M_{\mathrm{DM}}\left(h^{-1} \mathrm{Mpc}\right)<13.5$, at low $(<0.4)$ and high $(\sim 1)$ redshifts. Starikova et al. (2010) found that Chandra/ Bootes AGNs are located at the center of DM halos with $M>M_{\min }=4 \times 10^{12} h^{-1} M_{\odot}$. This mass estimate represents a threshold value, since they are assuming a halo occupation described by a step function (zero AGNs per halo/subhalo below

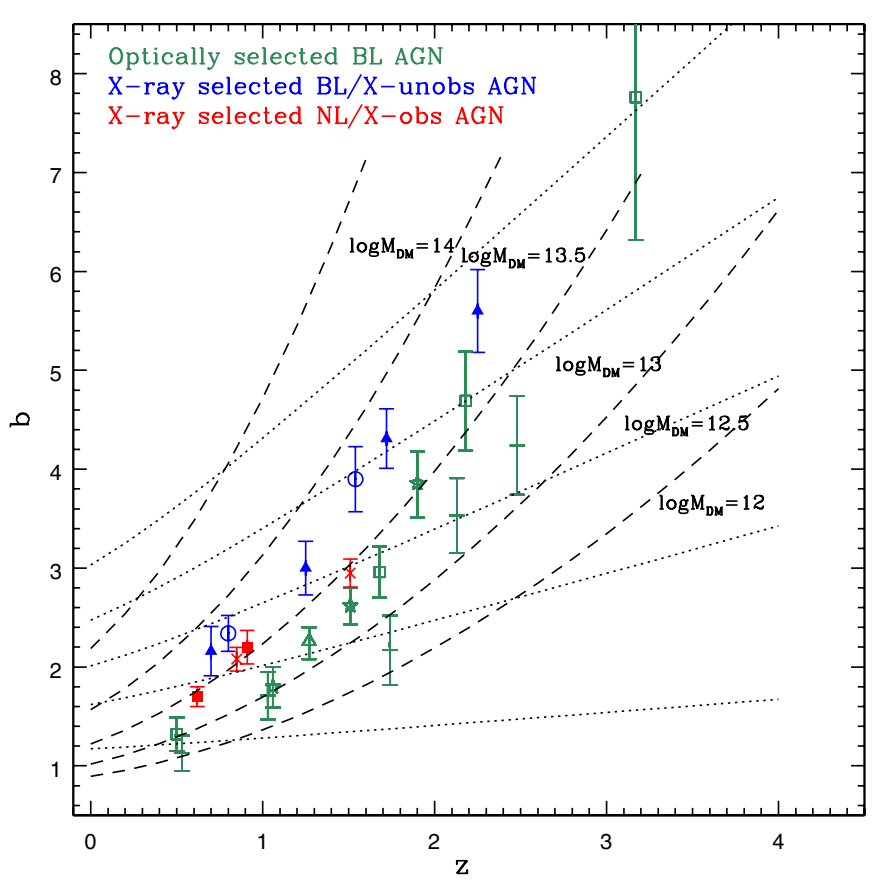

Figure 11. Bias parameter as a function of redshift for optically selected BL AGNs from previous works (Croom et al. 2005; green crosses), Porciani \& Norberg (2006; green stars), Shen et al. (2009; green open squares), Ross et al. (2009; green open triangle), and X-ray selected BL (blue triangles) and X-unobs (blue open-circles) AGNs and NL (red squares) and X-obs (red crosses) AGNs as estimated in this work. The dashed lines show the expected $b(z)$ of typical DM halo masses based on Sheth et al. (2001) and the dotted lines represent the passive evolution of the bias, as described in Fry et al. (1996). The bias of optically selected BL AGNs evolves with redshift following an evolution at constant halo mass, with a typical mass that remains practically in the range $\log M_{\mathrm{DM}} \sim 12.5-13 h^{-1} M_{\odot}$ at all redshifts $z<2.25$. X-ray selected BL/ $\mathrm{X}$-unobs AGNs reside in more massive DM halos at all redshifts $z<2.25$, according to a typical mass of the hosting halos constant over time in the range $\log M_{\mathrm{DM}} \sim 13-13.5 h^{-1} M_{\odot}$. The bias evolution of NL/X-obs AGNs seems to indicate that they reside in DM halos with masses of $\log M_{\mathrm{DM}} \sim 13 h^{-1} M_{\odot}$ constant at all $z<1.5$. These results suggest the picture that X-ray selected BL AGNs are triggered by secular processes as tidal disruption or disk instabilities instead of major mergers between gas-rich galaxies as confirmed by semianalytic models and observations for optically selected quasars.

(A color version of this figure is available in the online journal.)

$M_{\text {min }}$ and one above it). Our approach, in terms of HOD, is completely different. We assume a halo occupation described by the $\delta$-function, supported by the fact that AGNs only reside in massive halos (then the AGN HOD can be described by a narrow halo mass distribution at high mass values, but not by a step function).

Figure 10 shows the bias factors of X-ray selected AGNs (black), BL/X-unobs AGNs (blue), and NL/X-obs AGNs (red) as estimated in different surveys (according to the legend). Our results refer to the bias factors $b_{S 01}$ shown in Table 2, Column 5 . The dashed lines show the expected $b(z)$ assuming a constant typical DM halo mass $M_{\mathrm{DM}}$ based on Sheth et al. (2001).

The previous studies of Gilli et al. (2005) for the CDFN, Gilli et al. (2009), Mullis et al. (2004), Yang et al. (2006) for CLASXS AGNs suggest the scenario in which the typical DM halo mass hosting X-ray selected AGNs is $\log M_{\mathrm{DM}}\left(h^{-1} M_{\odot}\right) \sim 13.5$. The bias values measured in Gilli et al. (2005) on CDFS, in Hickox et al. (2009), Coil et al. (2009) and Yang et al. (2006), and in this work, correspond to a lower halo mass $\left(\log M_{\mathrm{DM}}\left(h^{-1} M_{\odot}\right) \sim 13\right)$. A possible explanation could be that, at a fixed redshift, the bias and then the mass of the hosting halo 
depends on the luminosity of the sample. The same explanation might be applied to the results for BL/X-unobs AGNs.

The bias estimates at $z<1$ for NL/X-obs AGNs in Cappelluti et al. (2010) and in this work seem to indicate that the mass of NL/X-obs AGNs hosting halos is $\log M_{\mathrm{DM}}\left(h^{-1} M_{\odot}\right) \sim 13$.

\subsection{Optically Selected versus X-Ray Selected AGNs}

We first found evidence of a redshift evolution of the bias factor of X-ray selected BL/X-unobs AGNs (Figure 11, blue data points) and NL/X-obs AGNs (red data points). The bias evolves with redshift at a constant average halo mass of $\log M_{0}\left(h^{-1} M_{\odot}\right) \sim 13.3$ for BL/X-unobs AGNs and $\log M_{0}\left(h^{-1} M_{\odot}\right) \sim 13$ for NL/X-obs AGNs at $z<2.25$ and $z<1.5$, respectively. Figure 11 shows the expected $b(z)$ assuming a constant typical DM halo mass based on Sheth et al. (2001) (dashed lines) and the so-called passive bias evolution (dotted lines; Fry et al. 1996). The observed bias evolution suggests an average halo mass of the hosting halos, constant over time in the range $\log M_{\mathrm{DM}}\left(h^{-1} M_{\odot}\right)=13-13.5$, instead of an evolution of the bias in a model in which objects are formed at a fixed time and their distribution evolves under the influence of gravity.

There have been several studies of the bias evolution of optical quasars with redshift as shown in Figure 11 (green data points), based on large survey samples such as 2QZ and SDSS (Croom et al. 2005; Porciani \& Norberg 2006; Shen et al. 2009; Ross et al. 2009). Since the quasar samples used in these clustering analyses are defined as spectroscopically identified quasars with at least one broad (FWHM > $1000 \mathrm{~km} \mathrm{~s}^{-1}$ ) emission line, we refer to them as optically selected BL AGNs.

All the previous studies infer the picture that the quasar bias evolves with redshift following a constant mass evolution, with an average mass that can vary in the range $\log M_{\mathrm{DM}}\left(h^{-1} M_{\odot}\right) \sim$ 12.5-13, which may be dependent on the AGN sample luminosity as already suggested for X-ray selected AGNs. The simplest interpretation according to the observed redshift evolution of the bias factors is that (1) X-ray selected AGNs, whether they are BL/X-unobs or NL/X-obs AGNs, inhabit DM halos with masses higher than the mass of optically selected quasar hosting halos in the range $z=0.5-2.25$; (2) X-ray selected BL/ $\mathrm{X}$-unobs AGNs reside in more massive halos compared to NL/ $\mathrm{X}$-obs AGNs for $z=0.6-1.6$, and the discrepancy between the bias factors of the two samples increases with $z$; (3) the AGN activity is a mass triggered phenomenon, and the different AGN evolutionary phases are associated with just the DM halo mass, irrespective of the redshift $z$.

\subsection{External versus Internal Triggering}

Major mergers of galaxies are one of the promising mechanisms suggested to be responsible for fueling quasars and, in particular, to be dominant for bright quasars at high redshifts. Models of major mergers appear to naturally produce many observed properties of quasars, such as the quasar luminosity density, the shape, and the evolution of the quasar luminosity function, and the large-scale quasar clustering as a function of $L$ and $z$ (Hopkins et al. 2008; Shen 2009; Shankar et al. 2009, 2010; Shankar 2010; Bonoli et al. 2009).

Clear evidence for a higher incidence of mergers is seen among quasars (Serber et al. 2006; Hopkins et al. 2006; Veilleux et al. 2009). Additionally a large fraction of luminous quasars at low redshift are associated with either morphologically disturbed objects (Canalizo \& Stockton 2001; Guyon et al.
2006), or early-type hosts with fine structures in their optical light distribution, indicative of past interactions (Canalizo et al. 2007; Bennert et al. 2008). In the local universe, for instance, the study of the environment of Swift/BAT Seyfert galaxies (Koss et al. 2010) appeared to show apparent mergers of $\sim 25 \%$ which suggests that AGN activity and merging are critically linked. Moreover, it is believed that major mergers dominate at high redshifts and bright luminosities (Hasinger 2008; Hopkins et al. 2006), while minor interaction or bar instabilities or minor tidal disruptions are important at low redshifts $(z \lesssim 1)$ and low luminosities $\left(L_{\mathrm{BOL}} \lesssim 10^{44} \mathrm{erg} \mathrm{s}^{-1}\right)$ (Hopkins \& Henquist 2009).

Our results on the bias evolution of X-ray selected BL/ X-unobs AGNs infer that these objects with $L_{\mathrm{BOL}} \sim 2 \times$ $10^{45} \mathrm{erg} \mathrm{s}^{-1}$ reside in massive DM halos of $M_{\mathrm{DM}} \sim 2 \times$ $10^{13} M_{\odot} h^{-1}$. Studies on BL AGNs in the COSMOS field (Merloni et al. 2010; Trump et al. 2011) suggest that our sample is characterized by $\mathrm{BH}$ masses in the range $M_{\mathrm{BH}}=10^{7}-10^{9} M_{\odot}$ and Eddington ratio $\lambda>0.01$. Optically selected quasars from large survey samples such as 2QZ and SDSS are highluminosity quasars $L_{\mathrm{BOL}} \gtrsim 10^{46} \mathrm{erg}^{-1}$ with $\mathrm{BH}$ masses in the range $M_{\mathrm{BH}}=10^{8}-10^{10} \stackrel{\sim}{M_{\odot}}$ and $\lambda>0.01$. Clustering analysis of optical quasars has shown that they reside in DM halos with $M_{\mathrm{DM}} \sim 10^{12} M_{\odot} h^{-1}$.

Figure 12 shows the predicted bias as a function of luminosity computed according to Shen (2009) at $z=2$. The theoretical model, which assumes a quasar phase triggered by a major merger predicts an increasing bias with luminosity and reproduces the previous results obtained for optical quasars at $1.8<z<2.2$ (Croom et al. 2005, green-crosses; Porciani \& Norberg 2006, green-star; Shen et al. 2009, green-open square; da Ângela et al. 2008, green-circles; Myers et al. 2007, greensquares). On the other hand, the model cannot reproduce the high bias factor found for X-ray selected COSMOS BL AGNs (blue triangle) and cannot explain why optically selected quasars characterized by higher bolometric luminosities compared to $\mathrm{X}$-ray selected COSMOS BL/X-unobs AGNs are found in less massive halos. These differences suggest a switch to a different dominant mechanism for AGN triggering.

Hopkins \& Hernquist (2006) introduced a model for the fueling of low-luminosity AGNs (Seyferts, with $L_{\mathrm{BOL}} \lesssim 10^{44}-10^{45} \mathrm{erg} \mathrm{s}^{-1}$ and $M_{\mathrm{BH}} \lesssim 10^{7} M_{\odot}$ ), which proposes AGNs triggered by random accretion of gas via internal, secular processes. The stochastic accretion model and the merger-driven activity are fundamentally different, the former being determined by stochastic encounters with a cold gas supply in a quiescent system and the latter by the violent torquing of cold gas throughout entire galaxies into the galaxy center in major mergers. Accretion of cold gas in quiescent systems can account for low-luminosity Seyferts but cannot explain the higher luminosities and the larger $\mathrm{BH}$ masses observed for $X M M$-COSMOS BL AGNs. The high Eddington ratios at masses in the range $M_{\mathrm{BH}} \sim 10^{8}-10^{9} M_{\odot}$ cannot be maintained through this mode of accretion.

Furthermore, this fueling mechanism predicts lower bias factors compared to the major merger picture for bright quasars, which is completely in disagreement with our results.

Fueling by stellar winds or hot gas accretion may represent yet a third qualitatively distinct mode of fueling. Ciotti \& Ostriker (2001a, 2001b) investigated the episodic AGN activity model in early-type galaxies, assuming at their center the presence of a massive $\mathrm{BH}$ growing with the accretion of matter and affecting the inflow through feedback. The duration of the single 


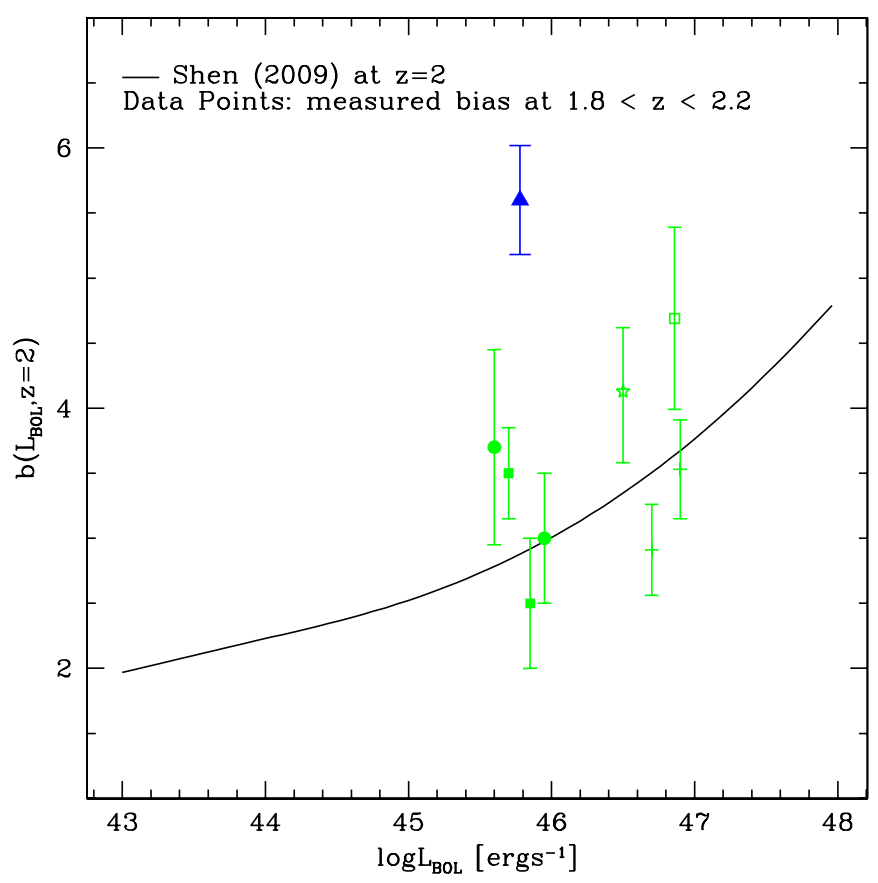

Figure 12. Predicted bias as a function of luminosity, computed according to Shen (2009) fixing $z=2$, compared to previous bias estimates at $1.8<z<2.2$, for optically selected BL AGNs and for XMM-COSMOS BL AGNs. Points are measurements from Croom et al. (2005, green crosses), Porciani \& Norberg (2006, green-star), Shen et al. (2009, green open square), da Ângela et al. (2008, green circles), Myers et al. (2007, green squares), and our result (blue triangle). For ease of comparison, all luminosities are converted to bolometric luminosities using the corrections from Hopkins et al. (2007). The theoretical model which assumes a quasar phase triggered by a major merger reproduces the results obtained for the bias of quasars, but cannot reproduce the high bias factors found for X-ray selected BL AGNs and cannot explain why optically selected quasars that have higher bolometric luminosities compared to COSMOS X-ray selected BL/X-unobs AGNs reside in more massive halos. These differences suggest a switch to a different dominant mechanism for AGN triggering, from major mergers between gas-rich galaxies to secular processes such as tidal disruptions or disk instabilities.

(A color version of this figure is available in the online journal.)

accretion event is extremely short but the maximum luminosities reached during the accretion events can be of the order of $L_{\mathrm{BOL}} \sim 10^{46}-10^{47} \mathrm{erg} \mathrm{s}^{-1}$, depending on the input parameters of the model. The central $\mathrm{BH}$ grows by episodic accretion up to a mass in the observed range $\left(M \sim 10^{8.5}-10^{9.5} M_{\odot}\right)$ of all giant ellipticals. On the other hand, the observational consequence of this model is that the duty cycle is very low, typically of the order of $10^{-2}$ to $10^{-3}$. This result implies a small fraction of giant ellipticals observed in an AGN phase, too low compared to the observed $10 \%$ of X-ray AGNs residing in massive galaxies.

In the AGN evolutionary model described in Hickox et al. (2009), optically bright quasars are hosted by ongoing disk galaxy mergers and immediately precede an optically faint X-ray AGN phase, which evolves into an early-type galaxy. Following this evolutionary sequence, NL/X-obs AGNs should be triggered in the first initial phase of vigorous star formation and obscured accretion, inhabiting halos with low typical masses of $\log M_{\mathrm{DM}}\left(h^{-1} M_{\odot}\right) \sim 12.5$, in disagreement with our results which suggest higher masses $\left(\sim 13 \mathrm{~h}^{-1} M_{\odot}\right)$. More massive host halos are also predicted in Hickox et al. (2011). They found that mid-infrared obscured quasars at $z \sim 1.2$ in the Boötes field inhabit halos with masses of $\log M_{\mathrm{DM}}\left(h^{-1} M_{\odot}\right)=13.3_{-0.4}^{+0.3}$. Following the model, an X-ray AGN phase immediately follows the quasar phase. Since DM halos grow and accumulate mass over time, X-ray AGNs reside in more massive DM halos with typical mass $\log M_{\mathrm{DM}}\left(h^{-1} M_{\odot}\right) \sim 13-13.5$. This model predicts that X-ray AGNs reside in more massive halos than QSO, but assumes a decline of the $\mathrm{BH}$ accretion rate from its peak in the quasar phase to $\dot{M} \lesssim 10^{-2} \dot{M_{\text {Edd }}}$ or lower, which is in disagreement with the high Eddington ratios found for XMMCOSMOS BL AGNs (Merloni et al. 2010; Trump et al. 2011).

A plausible scenario requires that high-luminosity quasars $\left(L_{\mathrm{BOL}}>10^{46} \mathrm{erg} \mathrm{s}^{-1}\right)$ are triggered by external processes such as major mergers between gas-rich galaxies with masses of the order of $M_{*} \sim 10^{10} M_{\odot}$. Instead, for BL AGNs with $L_{\mathrm{BOL}} \sim$ $2 \times 10^{45} \mathrm{erg} \mathrm{s}^{-1}$, internal mechanisms such as tidal disruptions or disk instabilities in massive galaxies $\left(M_{*} \sim 10^{11} M_{\odot}\right)$ might play a dominant role.

The morphology of the AGN host galaxies provides an important clue into the mechanism that triggers their current AGN activity. It was observed that many AGNs are not fueled by major mergers and only a small fraction of AGNs are associated with morphologically disturbed galaxies. Cisternas et al. (2011) analyzed a sample of X-ray selected AGN host galaxies and a matched control sample of inactive galaxies in the COSMOS field. They found that mergers and interactions involving AGN hosts are not dominant and occur no more frequently than for inactive galaxies. Over $55 \%$ of the studied AGN sample, which is characterized by $L_{\mathrm{BOL}} \sim 10^{45} \mathrm{erg} \mathrm{s}^{-1}$ and by masses of the host galaxies of $M_{*} \gtrsim 10^{10} M_{\odot}$, are hosted by disk-dominated galaxies. This high disk fraction means that the lack of disturbed morphologies observed among the AGN hosts cannot simply be due to a time lag between merger activity and X-ray visibility and suggests that secular fueling mechanisms can be highly efficient.

It was also suggested by Georgakakis et al. (2009) that bar instabilities and minor interactions are more efficient in producing luminous AGNs at $z \lesssim 1$ and not only Seyfert galaxies and low-luminosity AGNs as the Hopkins \& Hernquist (2006, 2009) model predicts. Moreover, several works on the AGN host galaxies by Dunlop et al. (2003), Grogin et al. (2005), Pierce et al. (2007), Gabor et al. (2009), Reichard et al. (2009), and Tal et al. (2009) show that the morphologies of the AGN host galaxies do not present a preference for merging systems.

At the redshift of our interest, recent findings of Schawinski et al. (2011) and Rosario et al. (2011), who examined a smaller sample of AGNs at $z \sim 2$ in the ERS-II region of the GOODS-South field, inferred that late-type morphologies are prevalent among the AGN hosts. The role that major galaxy mergers play in triggering AGN activity at $1.5<z<2.5$ was also studied in the CDF-S. D. D. Kocevski et al. (2011, in preparation) found that X-ray selected AGNs at $z \sim 2$ do not exhibit a significant excess of distorted morphologies while a large fraction reside in late-type galaxies. They also suggest that these late-type galaxies are fueled by the stochastic accretion of cold gas, possibly triggered by a disk instability or minor interaction.

We want to stress that our results by no means infer that mergers play no role in the AGN triggering. On the contrary, high-luminosity AGNs and probably a fraction of moderateluminosity AGNs in our sample might be fueled by mergers. In fact, given the complexity of AGN triggering, a proper selection of an AGN subsample, using, for instance, the luminosity, can help to test a particular model boosting the fraction of AGN host galaxies associated with morphologically disturbed galaxies.

Our work might extend the statement that for moderateluminosity X-ray selected BL AGNs secular processes might play a much larger role than major mergers up to $z \sim 2.2$, 
compared to the previous $z \lesssim 1$, even during the epoch of peak merger-driven accretion.

\section{CONCLUSIONS}

We have studied the redshift evolution of the bias factor of 593 XMM-COSMOS AGNs with spectroscopic redshifts $z<4$, extracted from the 0.5 to $2 \mathrm{keV} X$-ray image of the $2 \mathrm{deg}^{2}$ $X M M$-COSMOS field. We have described a new method to estimate the bias factor and the associated DM halo mass, which accounts for the growth of the structures over time and the sample variance. Key results can be summarized as follows.

1. We estimated the AGN bias factor $b_{S 01}=2.71 \pm 0.14$ at $\bar{z}=1.21$ which corresponds to a mass of DM halos hosting AGNs equal to $\log M_{0}\left(h^{-1} M_{\odot}\right)=13.10 \pm 0.10$.

2. We split the AGN sample into broad optical emission line AGNs (BL) and AGNs without optical broad emission lines (NL) and for each of them we considered a subset with $\bar{z}=0.6$ and we found that BL and NL AGNs present $b_{S 01}=$ $1.95 \pm 0.17$ and $b_{S 01}=1.62 \pm 0.15$, which correspond to masses equal to $\log M_{0}\left(h^{-1} M_{\odot}\right)=13.27 \pm 0.10$ and $12.97 \pm 0.07$, respectively.

3. We selected in the hard band a sample of X-ray unobscured and X-ray obscured AGNs according to the column density and we found that X-ray unobscured (X-ray obscured) AGNs inhabit DM halos with the same mass compared to BL (NL) AGNs with $\log M_{0}\left(h^{-1} M_{\odot}\right)=13.30 \pm 0.10$ $\left(\log M_{0}\left(h^{-1} M_{\odot}\right)=12.97 \pm 0.08\right)$.

4. We found evidence of a redshift evolution of the bias factors for the different AGN subsets, corresponding to a constant DM halo mass threshold which differs for each sample. XMM-COSMOS AGNs are hosted by DM halos with masses of $\log M_{0}=13.12 \pm 0.07\left(h^{-1} M_{\odot}\right)$ constant at all $z<2$, BL/X-ray unobscured AGNs reside in halos with masses $\log M_{0}=13.28 \pm 0.07\left(h^{-1} M_{\odot}\right)$ for $\bar{z}<2.25$ while $X M M$-COSMOS NL/X-ray obscured AGNs inhabit less massive halos $\log M_{0}=13.00 \pm 0.06\left(h^{-1} M_{\odot}\right)$, constant at all $\bar{z}<1.5$.

5. The observed bias evolution for $X M M-C O S M O S$ BL and NL AGNs at all $z<2.25$, suggests that the AGN activity is a mass triggered phenomenon and that different AGN evolutionary phases are associated with just the DM halo mass, irrespective of the redshift $z$.

6. The bias evolution of X-ray selected BL/X-ray unobscured AGNs corresponds to halo masses in the range $\log M_{\mathrm{DM}}\left(h^{-1} M_{\odot}\right) \sim 13-13.5$, typical of poor galaxy groups at all redshifts. Optically selected BL AGNs instead reside in lower density environments with constant halo masses in the range $\log M_{\mathrm{DM}}\left(h^{-1} M_{\odot}\right) \sim 12.5-13$ at all redshifts. This indicates that X-ray and optically selected AGNs do not inhabit the same DM halos.

7. The theoretical models that assume a quasar phase triggered by major mergers cannot reproduce the high bias factors and DM halo masses found for X-ray selected BL AGNs up to $z \sim 2.2$. Our results might suggest the statement that for moderate-luminosity X-ray selected BL AGNs secular processes such as tidal disruptions or disk instabilities play a much larger role than major mergers up to $z \sim 2.2$, compared to the previous $z \lesssim 1$.

We gratefully acknowledge the contributions of the entire COSMOS collaboration consisting of more than 100 scientists. More information on the COSMOS survey is available at http://www.astro.caltech.edu/ COSMOS. We thank the anonymous referee for carefully reading the manuscript and providing us with constructive remarks. V.A., G.H., and M.S. were supported by the German Deutsche Forschungsgemeinschaft, DFG Leibniz Prize (FKZ HA 1850/28-1).

\section{REFERENCES}

Adelman-McCarthy, J. K., et al. 2006, ApJS, 162, 38

Bennert, N., Canalizo, G., Jungwiert, B., Stockton, A., Schweizer, F., Peng, C.Y., \& Lacy, M. 2008, ApJ, 677, 846

Bonoli, S., Marulli, F., Springel, V., White, S. D. M., Branchini, E., \& Moscardini, L. 2009, MNRAS, 396, 423

Brusa, M., et al. 2010, ApJ, 716, 348

Canalizo, G., Bennert, N., Jungwiert, B., Stockton, A., Schweizer, F., Lacy, M., \& Peng, C. 2007, ApJ, 669, 801

Canalizo, G., \& Stockton, A. 2001, ApJ, 555, 719

Cappelluti, N., et al. 2007, ApJS, 172, 341

Cappelluti, N., et al. 2009, A\&A, 497, 635

Cappelluti, N., et al. 2010, ApJ, 716, 209

Ciotti, L., \& Ostriker, J. P. 2001a, ApJ, 487, L105

Ciotti, L., \& Ostriker, J. P. 2001b, ApJ, 551, 131

Cisterans, M., et al. 2011, ApJ, 726, 57

Coil, A. L., Hennawi, J. F., Newman, J. A., Cooper, M. C., \& Davis, M. 2007, ApJ, 654, 115

Coil, A. L., et al. 2008, ApJ, 672, 153

Coil, A. L., et al. 2009, ApJ, 701, 1484

Croom, S. M., et al. 2005, MNRAS, 356, 415

da Ângela, J., et al. 2008, MNRAS, 383, 565

Davis, M., \& Peebles, P. J. E. 1983, ApJ, 267, 465

Dunlop, J. S., McLure, R. J., Kukula, M. J., Baum, S. A., O’Dea, C. P., \& Hughes, D. H. 2003, MNRAS, 340, 1095

Eisenstein, D. J., \& Hu, W. 1999, ApJ, 511, 5

Elvis, M., et al. 2009, ApJS, 184, 158

Faltenbacher, A., Finoguenov, A., \& Drory, N. 2010, ApJ, 712, 484

Fry, J. N. 1996, ApJ, 461, 65

Gabor, J. M., et al. 2009, ApJ, 691, 705

Georgakakis, A., et al. 2009, MNRAS, 397, 623

Gilli, R., et al. 2005, A\&A, 430, 811

Gilli, R., et al. 2009, A\&A, 494, 33

Grogin, N. A., et al. 2005, ApJ, 627, 97

Guyon, O., Sanders, D. B., \& Stockton, A. 2006, ApJS, 166, 89

Hamana, T., Yoshida, N., \& Suto, Y. 2002, ApJ, 568, 455

Hasinger, G. 2008, A\&A, 490, 905

Hasinger, G., et al. 2007, ApJS, 172, 29

Hickox, R. C., Jones, C., \& Forman, W. R. 2009, ApJ, 696, 891

Hickox, R. C., et al. 2011, ApJ, 731, 117

Hopkins, P. F., \& Hernquist, L. 2006, ApJS, 166, 1

Hopkins, P. F., \& Hernquist, L. 2009, ApJ, 694, 599

Hopkins, P. F., Hernquist, L., Cox, T. J., Di Matteo, T., Robertson, B., \& Springel, V. 2006, ApJS, 163, 1

Hopkins, P. F., Hernquist, L., Cox, T. J., \& Keres, D. 2008, ApJS, 175, 356

Hopkins, P. F., Richards, G. T., \& Henquist, L. 2007, ApJ, 654, 731

Ilbert, O., et al. 2009, ApJ, 690, 1236

Koss, M., Mushotzky, R., Veilleux, S., \& Winter, L. 2010, ApJ, 716, 125

Krumpe, M., Miyaji, T., \& Coil, A. L. 2010, ApJ, 713, 558

Landy, S. D., \& Szalay, A. S. 1993, ApJ, 412, 64

Le Floc'h, E., et al. 2009, ApJ, 703, 222

Li, C., Kauffmann, G., Wang, L., White, S. D. M., Heckman, T. M., \& Jing, Y. P. 2006, MNRAS, 373, 457

Lilly, S. J., et al. 2007, ApJS, 172, 70

Lilly, S. J., et al. 2009, ApJS, 184, 218

Martini, P., Sivakoff, G. R., \& Mulchaey, J. S. 2009, ApJ, 701, 66

McCracken, H., et al. 2010, ApJ, 708, 202

Merloni, A., et al. 2010, ApJ, 708, 137

Miyaji, T., Krumpe, M., Coil, A. L., \& Aceves, H. 2011, ApJ, 726, 83

Miyaji, T., et al. 2007, ApJS, 172, 396

Mo, H. J., \& White, S. D. M. 1996, MNRAS, 282, 347

Mullis, C. R., Henry, J. P., Gioia, I. M., Bhringer, H., Briel, U. G., Voges, W., \& Huchra, J. P. 2004, ApJ, 617, 192

Myers, A. D., Brunner, R. J., Richards, G. T., Nichol, R. C., Schneider, D. P., \& Bahcall, N. A. 2007, ApJ, 658, 99

Peebles, P. J. E. 1980, The Large Scale Structure of the Universe (Princeton, NJ: Princeton Univ. Press)

Pierce, C. M., et al. 2007, ApJ, 660, 19 
Porciani, C., \& Norberg, P. 2006, MNRAS, 371, 1824

Prescott, M. K. M., Impey, C. D., Cool, R. J., \& Scoville, N. Z. 2006, ApJ, 644, 100

Reichard, T. A., Heckmas, T. M., Rudnick, G., Brinchmann, J., Kauffmann, G., \& Wild, V. 2009, ApJ, 691, 1005

Rosario, D. J., McGurk, R. C., Max, C. E., Shields, G. A., \& Smith, K. L. 2011, arXiv:1102.1733R

Ross, N. P., et al. 2009, ApJ, 697, 1634

Salvato, M., et al. 2009, ApJ, 690, 1250

Sanders, D. B, et al. 2007, ApJS, 172, 86

Schawinski, K., Treister, E., Urry, C. M., Cardamone, C. N., Simmons, B., \& Yi, S. K. 2011, ApJ, 727, 31

Scoville, N., et al. 2007, ApJS, 172, 38

Serber, W., Bahcall, N., Menard, B., \& Richards, G. 2006, ApJ, 643, 68

Shankar, F. 2010, in IAU Symp. 267, Co-evolution of Central Black Holes and Galaxies, ed. B. Peterson, R. Somerville, \& T. Storchi-Bergmann (Cambridge: Cambridge Univ. Press), 248

Shankar, F., Weinberg, D. H., \& Miralda-Escudé, J. 2009, ApJ, 690, 20
Shankar, F., et al. 2010, ApJ, 718, 231

Shen, Y. 2009, ApJ, 704, 89

Shen, Y., et al. 2009, ApJ, 697, 1656

Sheth, R. K., Mo, H. J., \& Tormen, G. 2001, MNRAS, 323, 1

Sheth, R. K., \& Tormen, G. 1999, MNRAS, 308, 119

Silverman, J. D., Kovač, K., \& Knobel, C. 2009, ApJ, 695, 171

Starikova, S., et al. 2010, arXiv:1010.1577S

Tal, T., van Dokkum, P. G., Nelan, J., \& Bezanson, R. 2009, ApJ, 138, 1417

Taniguchi, Y., et al. 2007, ApJS, 172, 9

Tinker, J. L., Weinberg, D. H., Zheng, Z., \& Zehavi, I. 2005, ApJ, 631, 41

Trump, J. R., et al. 2007, ApJS, 172, 383

Trump, J. R., et al. 2011, ApJ, 733, 60

van den Bosch, F. C. 2002, MNRAS, 331, 98

Veilleux, S., et al. 2009, ApJ, 701, 587

Yang, Y., Mushotzky, R. F., Barger, A. J., \& Cowie, L. L. 2006, ApJ, 645, 68 Zamojski, M. A., et al. 2007, ApJS, 172, 468

Zehavi, I., et al. 2005, ApJ, 621, 22 